\title{
THE ELEMENTARY THEORY OF LARGE FIELDS OF TOTALLY S-ADIC NUMBERS
}

\author{
ARNO FEHM
}

\begin{abstract}
We analyze the elementary theory of certain fields $K^{\mathfrak{S}}(\boldsymbol{\sigma})$ of totally $\mathfrak{S}$ adic algebraic numbers that were introduced and studied by Geyer-Jarden and HaranJarden-Pop. In particular, we provide an axiomatization of these theories and prove their decidability, thereby giving a common generalization of classical decidability results of Jarden-Kiehne, Fried-Haran-Völklein and Ershov.
\end{abstract}

\section{INTRODUCTION}

Let $\mathfrak{S}$ be a finite set of absolute values on a number field $K$. By $K^{\mathfrak{S}}$ we denote the field of totally $\mathfrak{S}$-adic numbers - the maximal Galois extension of $K$ in which the elements of $\mathfrak{S}$ are totally split. For an integer $e \geq 0$ and an $e$-tuple $\boldsymbol{\sigma}=\left(\sigma_{1}, \ldots, \sigma_{e}\right) \in \operatorname{Gal}(K)^{e}$ of elements of the absolute Galois group of $K$, we let $K^{\mathfrak{S}}(\boldsymbol{\sigma})$ be the fixed field of the group $\left\langle\sigma_{1}, \ldots, \sigma_{e}\right\rangle \leq \operatorname{Gal}(K)$ inside $K^{\mathfrak{S}}$.

These fields $K^{\mathfrak{S}}(\boldsymbol{\sigma})$ were studied by Jarden-Razon [JR98, Geyer-Jarden [GJ02, and recently in a series of papers by Haran-Jarden-Pop [HJP09], [HJP12]. In particular, these authors prove that for almost all $\boldsymbol{\sigma} \in \operatorname{Gal}(K)^{e}$, in the sense of Haar measure on the compact group $\mathrm{Gal}(K)^{e}$, the field $K^{\mathfrak{S}}(\boldsymbol{\sigma})$ satisfies a local-global principle for rational points on varieties, and its absolute Galois group has a nice description as a free product of local factors.

Combining these results we are able to give an axiomatization of the theory $T_{\text {almost, }, e}$ of first-order sentences in the language of rings that hold in almost all $K^{\mathfrak{S}}(\boldsymbol{\sigma})$ (Theorem 10.11) and we prove the decidability of this theory (Theorem 11.12):

Theorem 1.1. Let $\mathfrak{S}$ be a finite set of absolute values on a number field $K$, and let $e \geq 0$. Then the first-order theory $T_{\text {almost, }, \mathfrak{S}, e}$ of almost all $K^{\mathfrak{S}}(\boldsymbol{\sigma}), \boldsymbol{\sigma} \in \operatorname{Gal}(K)^{e}$, is decidable.

This theorem is a common generalization of classical decidability results of JardenKiehne [JK75] (the case $\mathfrak{S}=\emptyset$ ), Fried-Haran-Völklein [FHV94] (the case $K=\mathbb{Q}, e=0$ and $\mathfrak{S}$ consisting only of the archimedean absolute value), and Ershov [Ers96b] (the case $K=\mathbb{Q}, e=0$ and $\mathfrak{S}$ consisting only of $p$-adic absolute values).

In fact, we prove a more general and stronger statement, see Theorem 11.12. The main part of the proof consists of an analysis of the absolute Galois group of $K^{\mathfrak{S}}(\boldsymbol{\sigma})$ together with local data, and the model theory of such structures.

\section{Preliminaries on PROFinite Groups AND SPACES}

We assume that the reader is familiar with the basic theory of profinite groups, as presented in [FJ08, Ch. 1], [RZ00, Ch. 2].

We always consider profinite groups as topological groups, so in particular homomorphisms between profinite groups are continuous group homomorphisms. By $H \leq G$ (resp. $H \triangleleft G$ ) we indicate that $H$ is a closed (resp. normal closed) subgroup of $G$. If $X \subseteq G$, we denote by $\langle X\rangle$ the closed subgroup generated by $X$ in $G$. We use the symbol 1 to denote both the unit element of $G$, and the trivial subgroup $\{1\} \leq G$. 
In the category of profinite groups, direct products, inverse limits and fibre products exist, FJ08, 22.2.1]. For the notion of the $\operatorname{rank} \operatorname{rk}(G)$ of a profinite group $G$ and the notion of a free profinite group see [FJ08, Ch. 17]. We denote by $\hat{F}_{e}$ the free profinite group of rank $e$.

Lemma 2.1. Let $\pi: G \rightarrow H$ be an epimorphism of profinite groups. Let $e \geq 0$, let $N \triangleleft G$ be a closed normal subgroup with $\operatorname{rk}(G / N) \leq e$, and let $h_{1}, \ldots, h_{e} \in H$ such that $H=\left\langle h_{1}, \ldots, h_{e}, \pi(N)\right\rangle$. Then there exist $g_{1}, \ldots, g_{e} \in G$ such that $G=\left\langle g_{1}, \ldots, g_{e}, N\right\rangle$ and $\pi\left(g_{i}\right)=h_{i}, i=1, \ldots, e$.

Proof. Let $\bar{G}=G / N, \bar{H}=H / \pi(N)$, and let $\bar{\pi}: \bar{G} \rightarrow \bar{H}$ be the induced epimorphism. Then $\bar{H}=\left\langle\bar{h}_{1}, \ldots, \bar{h}_{e}\right\rangle$, so Gaschütz' lemma [FJ08, 17.7.2] implies that there are $g_{1}, \ldots, g_{e} \in G$ such that $\bar{G}=\left\langle\bar{g}_{1}, \ldots, \bar{g}_{e}\right\rangle$ and $\bar{\pi}\left(\bar{g}_{i}\right)=\bar{h}_{i}, i=1, \ldots, e$. So, $G=\left\langle g_{1}, \ldots, g_{e}, N\right\rangle$ and there are $n_{1}, \ldots, n_{e} \in N$ such that $\pi\left(g_{i}\right)=h_{i} \pi\left(n_{i}\right), i=1, \ldots, e$. Thus, setting $g_{i}^{\prime}=g_{i} n_{i}^{-1}$, we have $G=\left\langle g_{1}^{\prime}, \ldots, g_{e}^{\prime}, N\right\rangle$ and $\pi\left(g_{i}^{\prime}\right)=h_{i}, i=1, \ldots, e$.

A profinite space is a totally disconnected compact Hausdorff space. Profinite spaces can be characterized as inverse limits of finite discrete spaces, or as zero-dimensional compact Hausdorff spaces, [RZ00, 1.1.12]. Any product and any finite coproduct (i.e. direct sum) of profinite spaces is a profinite space, and a subspace of a profinite space is profinite if and only if it is closed. Since profinite spaces are compact Hausdorff, any continuous map between profinite spaces is closed, and any continuous bijection of profinite spaces is a homeomorphism.

\section{Group Piles}

The notion of group piles was introduced in [HJP09] to enrich profinite groups with extra local data. We recall this notion and extend it. Our main innovation is the introduction of a certain quotient $\overline{\mathbf{G}}$ that measures the failure of a deficient group pile $\mathbf{G}$ to be selfgenerated.

Fix a finite set $\mathfrak{S}$ not containing 0 and let $e \geq 0$.

Definition 3.1. Let $G=\lim _{N} G / N$ be a profinite group, where $N$ runs over all open normal subgroups of $G$. Then the set $\operatorname{Subgr}(G)$ of all closed subgroups of $G$ is equipped with a profinite topology, induced by $\operatorname{Subgr}(G)=\lim _{N} \operatorname{Subgr}(G / N)$. The group $G$ acts continuously on $\operatorname{Subgr}(G)$ by conjugation. A homomorphism $\alpha$ : $G \rightarrow H$ of profinite groups induces a map $\operatorname{Subgr}(\alpha): \operatorname{Subgr}(G) \rightarrow \operatorname{Subgr}(H)$ given by $\Gamma \mapsto \alpha(\Gamma)$.

Lemma 3.2. The map Subgr is a covariant functor from the category of profinite groups (with homomorphisms) to the category of profinite spaces (with continuous maps).

Proof. It is easy to check that if $\alpha: G \rightarrow H$ is a homomorphism of profinite groups, then the induced map $\operatorname{Subgr}(G) \rightarrow \operatorname{Subgr}(H)$ is continuous.

Lemma 3.3. If $H$ is a closed subgroup of a profinite group $G$, then $\operatorname{Subgr}(H)$ is a closed subspace of $\operatorname{Subgr}(G)$.

Proof. By Lemma 3.2, the inclusion $\iota: \operatorname{Subgr}(H) \rightarrow \operatorname{Subgr}(G)$ is continuous. Since both spaces are compact Hausdorff, $\iota$ is closed, and thus a topological embedding.

Definition 3.4. A group pile is a structure $\mathbf{G}=\left(G, \mathcal{G}_{0}, \mathcal{G}_{\mathfrak{p}}\right)_{\mathfrak{p} \in \mathfrak{S}}$ consisting of

(1) a profinite group $G$,

(2) a nonempty $G$-invariant closed subset $\mathcal{G}_{0} \subseteq \operatorname{Subgr}(G)$ such that the elements of $\mathcal{G}_{0}$ are pairwise conjugate in $G$, and 
(3) a $G$-invariant closed subset $\mathcal{G}_{\mathfrak{p}} \subseteq \operatorname{Subgr}(G)$ for each $\mathfrak{p} \in \mathfrak{S}$.

The order resp. rank of $\mathbf{G}$ is the order resp. rank of $G$. A finite group pile is a group pile of finite order. Let $\mathcal{G}=\bigcup_{\mathfrak{p} \in \mathfrak{S}} \mathcal{G}_{\mathfrak{p}}$. We call $\mathbf{G}$ self-generated if there exists $G_{0} \in \mathcal{G}_{0}$ such that $G=\left\langle G_{0}, \mathcal{G}\right\rangle$, i.e. $G$ is generated by $G_{0}$ and the groups in $\mathcal{G}_{\mathfrak{p}}, \mathfrak{p} \in \mathfrak{S}$. It is called bare if $\mathcal{G}=\{1\}$, and deficient if $\mathcal{G}_{0}=\{1\}$. The deficient reduct of $\mathbf{G}$ is

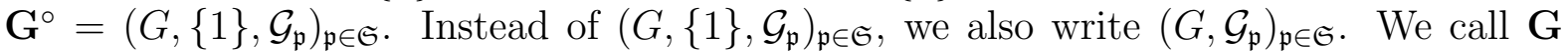
separated if the sets $\mathcal{G}_{\mathfrak{p}}, \mathfrak{p} \in\{0\} \cup \mathfrak{S}$, are disjoint, and reduced if there are no non-trivial inclusions among the elements of $\mathcal{G}$.

Remark 3.5. Note that if $\mathbf{G}$ is self-generated, then $G=\left\langle G_{0}, \mathcal{G}\right\rangle$ for any $G_{0} \in \mathcal{G}_{0}$. Condition (2) says that $\mathcal{G}_{0}$ consists of a single $G$-orbit in $\operatorname{Subgr}(G)$, i.e. there exists $G_{0} \in \mathcal{G}_{0}$ such that $\mathcal{G}_{0}=\left(G_{0}\right)^{G}:=\left\{\left(G_{0}\right)^{g}: g \in G\right\}$. Hence, our notion of group piles coincides with the group piles of [HJP09], except for a small difference in notation concerning $\mathcal{G}_{0}$. The notion of group piles is also related to the ' $\Delta^{*}$-groups' in [Ers95], [Ers96a], and [Ers99.

Definition 3.6. A homomorphism of group piles

$$
f:\left(G, \mathcal{G}_{0}, \mathcal{G}_{\mathfrak{p}}\right)_{\mathfrak{p} \in \mathfrak{S}} \rightarrow\left(H, \mathcal{H}_{0}, \mathcal{H}_{\mathfrak{p}}\right)_{\mathfrak{p} \in \mathfrak{S}}
$$

is a homomorphism of profinite groups $f: G \rightarrow H$ such that $f\left(\mathcal{G}_{\mathfrak{p}}\right) \subseteq \mathcal{H}_{\mathfrak{p}}$ for each $\mathfrak{p} \in$ $\{0\} \cup \mathfrak{S}$. It is an epimorphism if $f: G \rightarrow H$ is surjective and $f\left(\mathcal{G}_{\mathfrak{p}}\right)=\mathcal{H}_{\mathfrak{p}}$ for each $\mathfrak{p} \in\{0\} \cup \mathfrak{S}$. It is an isomorphism if in addition $f: G \rightarrow H$ is an isomorphism. The homomorphism $f$ is called rigid if $\left.f\right|_{\Gamma}$ is injective for each $\Gamma \in \mathcal{G}$. If $N$ is a closed normal subgroup of $G$, define the quotient $\mathbf{G} / N=\left(G / N, \mathcal{G}_{0, N}, \mathcal{G}_{\mathfrak{p}, N}\right)_{\mathfrak{p} \in \mathfrak{S}}$ by $\mathcal{G}_{\mathfrak{p}, N}=$ $\left\{\Gamma N / N: \Gamma \in \mathcal{G}_{\mathfrak{p}}\right\} \subseteq \operatorname{Subgr}(G / N)$. This is again a group pile, the quotient map $G \rightarrow G / N$ extends to an epimorphism of group piles $\mathbf{G} \rightarrow \mathbf{G} / N$, and every epimorphism of group piles is of this form.

Remark 3.7. We identify the category of bare deficient group piles (with homomorphisms) with the category of profinite groups (with homomorphisms) via the forgetful functor $\left(G, \mathcal{G}_{0}, \mathcal{G}_{\mathfrak{p}}\right)_{\mathfrak{p} \in \mathfrak{S}} \mapsto G$.

Lemma 3.8. In the category of group piles with epimorphisms, inverse limits exist.

Proof. For a directed set $I$ and an inverse family $\mathbf{G}_{i}=\left(G_{i}, \mathcal{G}_{i, 0}, \mathcal{G}_{i, \mathfrak{p}}\right)_{\mathfrak{p} \in \mathfrak{S}}, i \in I$, of group piles, $\mathbf{G}=\left(G, \mathcal{G}_{0}, \mathcal{G}_{\mathfrak{p}}\right)_{\mathfrak{p} \in \mathfrak{S}}$ with $G:=\lim _{i \in I} G_{i}$ and $\mathcal{G}_{\mathfrak{p}}:=\lim _{i \in I} \mathcal{G}_{i, \mathfrak{p}} \subseteq \operatorname{Subgr}(G), \mathfrak{p} \in \mathfrak{S} \cup$ $\{0\}$, is an inverse limit.

Definition 3.9. For a group pile $\mathbf{G}=\left(G, \mathcal{G}_{0}, \mathcal{G}_{\mathfrak{p}}\right)_{\mathfrak{p} \in \mathfrak{S}}$ let $G^{\prime}:=\langle\mathcal{G}\rangle$ be the closed subgroup generated by the subgroups in $\mathcal{G}_{\mathfrak{p}}, \mathfrak{p} \in \mathfrak{S}$. Let $\mathbf{G}^{\prime}:=\left(G^{\prime}, \mathcal{G}_{\mathfrak{p}}\right)_{\mathfrak{p} \in \mathfrak{S}}$ and $\overline{\mathbf{G}}:=\mathbf{G} / G^{\prime}$. We say that $\mathbf{G}$ is $e$-generated if $\operatorname{rk}(\overline{\mathbf{G}}) \leq e$, and $e$-bounded if $\mathbf{G}$ is self-generated and $\operatorname{rk}\left(G_{0}\right) \leq e$ for all $G_{0} \in \mathcal{G}_{0}$.

Lemma 3.10. $\mathbf{G}^{\prime}$ is a self-generated and deficient group pile, and $\overline{\mathbf{G}}$ is a bare group pile.

Proof. By Lemma 3.3, $\mathbf{G}^{\prime}$ is a group pile. The other claims are obvious.

Lemma 3.11. If $\varphi: \mathbf{G} \rightarrow \mathbf{H}$ is an epimorphism of group piles, then $\varphi\left(G^{\prime}\right)=H^{\prime}$, so $\varphi$ induces epimorphisms $\varphi^{\prime}: \mathbf{G}^{\prime} \rightarrow \mathbf{H}^{\prime}$ and $\bar{\varphi}: \overline{\mathbf{G}} \rightarrow \overline{\mathbf{H}}$.

Proof. By definition of an epimorphism of group piles, $\varphi(\mathcal{G})=\mathcal{H}$. Hence, since $\varphi$ is continuous and closed, $\varphi(\langle\mathcal{G}\rangle)=\langle\mathcal{H}\rangle$, as claimed.

Lemma 3.12. The map $\mathbf{G} \mapsto \mathbf{G}^{\prime}$ (resp. $\mathbf{G} \mapsto \overline{\mathbf{G}}$ ) is a covariant functor from the category of group piles with epimorphisms to the category of self-generated deficient group piles with epimorphisms (resp. the category of bare group piles with epimorphisms). 
Proof. This follows from Lemma 3.11.

Lemma 3.13. Let $\mathbf{G}=\left(G, \mathcal{G}_{\mathfrak{p}}\right)_{\mathfrak{p} \in \mathfrak{S}}$ be a deficient group pile and $\mathbf{A}=A$ a bare deficient group pile. Then the map $\varphi \mapsto \bar{\varphi}$ gives a bijection between the epimorphisms from $\mathbf{G}$ to A and the epimorphisms from $\bar{G}$ to $A$.

Proof. If $\varphi: \mathbf{G} \rightarrow \mathbf{A}$ is an epimorphism, then $\bar{\varphi}: \overline{\mathbf{G}} \rightarrow \overline{\mathbf{A}}=\mathbf{A}$ is an epimorphism. Conversely, given an epimorphism $\bar{\varphi}: \bar{G} \rightarrow A$, the composition $\bar{\varphi} \circ \pi: \mathbf{G} \rightarrow \mathbf{A}$, where $\pi: \mathbf{G} \rightarrow \overline{\mathbf{G}}$ is the quotient map, is an epimorphism. These two operations are inverse to each other.

Remark 3.14. Note that a deficient group pile is self-generated if and only if it is 0generated. Every $e$-bounded group pile is $e$-generated.

Lemma 3.15. Let $\varphi: \mathbf{G} \rightarrow \mathbf{H}$ be an epimorphism of group piles. If $\mathbf{G}$ is e-generated, then $\mathbf{H}$ is e-generated. If $\mathbf{G}$ is e-bounded, then $\mathbf{H}$ is e-bounded.

Proof. The induced map $\bar{\varphi}: \overline{\mathbf{G}} \rightarrow \overline{\mathbf{H}}$ is an epimorphism by Lemma 3.12 , so $\operatorname{rk}(\bar{H}) \leq \operatorname{rk}(\bar{G})$. If $\mathbf{G}$ is self-generated, then also $\mathbf{H}$ is self-generated. Since $\varphi\left(\mathcal{G}_{0}\right)=\mathcal{H}_{0}$, for $H_{0} \in \mathcal{H}_{0}$ there exists $G_{0} \in \mathcal{G}_{0}$ with $\varphi\left(G_{0}\right)=H_{0}$ and thus $\operatorname{rk}\left(H_{0}\right) \leq \operatorname{rk}\left(G_{0}\right)$.

Proposition 3.16. A deficient group pile $\mathbf{G}$ is e-generated if and only if every finite quotient of $\mathbf{G}$ is e-generated.

Proof. If $\mathbf{G}$ is e-generated, then every finite quotient of $\mathbf{G}$ is $e$-generated by Lemma 3.15. Conversely, suppose that $\mathbf{G}$ is not e-generated. Then there is an epimorphism $\bar{G} \rightarrow A$ onto a finite group $A$ with $\operatorname{rk}(A)>e$ (see for example [RZ00, 2.5.3]), so $A$ is a finite quotient of $\mathbf{G}$ (Lemma 3.13) which is not $e$-generated.

Lemma 3.17. Let $\mathbf{A}$ be an e-bounded self-generated group pile and let $\tilde{\mathbf{B}}=\left(B, \mathcal{B}_{\mathfrak{p}}\right)_{\mathfrak{p} \in \mathfrak{S}}$ be an e-generated deficient group pile. For every epimorphism $\pi: \tilde{\mathbf{B}} \rightarrow \mathbf{A}^{\circ}$ there exists an ebounded self-generated group pile $\mathbf{B}$ with $\mathbf{B}^{\circ}=\tilde{\mathbf{B}}$ such that $\pi: \mathbf{B} \rightarrow \mathbf{A}$ is an epimorphism.

Proof. Let $A_{0} \in \mathcal{A}_{0}$ and choose $a_{1}, \ldots, a_{e} \in A$ with $A_{0}=\left\langle a_{1}, \ldots, a_{e}\right\rangle$. By Lemma 3.11, $A=\left\langle a_{1}, \ldots, a_{e}, A^{\prime}\right\rangle$ and $A^{\prime}=\pi\left(B^{\prime}\right)$, so Lemma 2.1 gives $b_{1}, \ldots, b_{e} \in B$ with $B=$ $\left\langle b_{1}, \ldots, b_{e}, B^{\prime}\right\rangle$ and $\pi\left(b_{i}\right)=a_{i}$. Let $B_{0}=\left\langle b_{1}, \ldots, b_{e}\right\rangle$ and $\mathbf{B}=\left(B,\left(B_{0}\right)^{B}, \mathcal{B}_{\mathfrak{p}}\right)_{\mathfrak{p} \in \mathfrak{S}}$. Then $\mathbf{B}$ is $e$-bounded and $\pi: \mathbf{B} \rightarrow \mathbf{A}$ is an epimorphism.

\section{Embedding Problems}

We recall the notion of embedding problems for group piles from [HJP09, §4] and rephrase some results in terms of $e$-bounded group piles.

Definition 4.1. Let $\mathbf{G}$ be a group pile. An embedding problem for $\mathbf{G}$ is a pair

$$
E P=(\varphi: \mathbf{G} \rightarrow \mathbf{A}, \alpha: \mathbf{B} \rightarrow \mathbf{A})
$$

of epimorphisms of group piles. It is called finite, self-generated, $e$-generated, $e$ bounded, deficient, or bare, if $\mathbf{B}$ has this property. It is called rigid if $\alpha$ is rigid. A solution of the embedding problem $(\varphi, \alpha)$ is an epimorphism $\gamma: \mathbf{G} \rightarrow \mathbf{B}$ such that $\alpha \circ \gamma=\varphi$. The embedding problem $E P$ is locally solvable if, writing $\mathbf{G}=\left(G, \mathcal{G}_{0}, \mathcal{G}_{\mathfrak{p}}\right)_{\mathfrak{p} \in \mathfrak{S}}$ and $\mathbf{B}=\left(B, \mathcal{B}_{0}, \mathcal{B}_{\mathfrak{p}}\right)_{\mathfrak{p} \in \mathfrak{S}}$, the following holds for every $\mathfrak{p} \in\{0\} \cup \mathfrak{S}$ :

For every $\Gamma \in \mathcal{G}_{\mathfrak{p}}$ there is a $\Delta \in \mathcal{B}_{\mathfrak{p}}$, and for every $\Delta \in \mathcal{B}_{\mathfrak{p}}$ there is a $\Gamma \in \mathcal{G}_{\mathfrak{p}}$, such that there exists an epimorphism $\gamma_{\Gamma}: \Gamma \rightarrow \Delta$ with $\alpha \circ \gamma_{\Gamma}=\left.\varphi\right|_{\Gamma}$. 
Lemma 4.2. If there exists $G_{0} \in \mathcal{G}_{0}$ and $B_{0} \in \mathcal{B}_{0}$ and an epimorphism $\gamma_{0}: G_{0} \rightarrow B_{0}$ with $\alpha \circ \gamma_{0}=\left.\varphi\right|_{G_{0}}$, then $(\underline{L S})$ holds for $\mathfrak{p}=0$.

Proof. If $g \in G$ and $\Gamma=\left(G_{0}\right)^{g} \in \mathcal{G}_{0}$, choose $b \in B$ with $\alpha(b)=\varphi(g)$, and let $\Delta=\left(B_{0}\right)^{b}$. If $b \in B$ and $\Delta=\left(B_{0}\right)^{b} \in \mathcal{B}_{0}$, choose $g \in G$ with $\varphi(g)=\alpha(b)$, and let $\Gamma=\left(G_{0}\right)^{g}$. Define $\gamma_{\Gamma}: \Gamma \rightarrow \Delta$ by $\gamma_{\Gamma}(x)=\gamma_{0}\left(x^{g^{-1}}\right)^{b}$. Then $\alpha\left(\gamma_{\Gamma}(x)\right)=\varphi\left(x^{g^{-1}}\right)^{\varphi(g)}=\varphi(x)$ for all $x \in \Gamma$.

Lemma 4.3. Every rigid embedding problem satisfies $(L S)$ for every $\mathfrak{p} \in \mathfrak{S}$.

Proof. Suppose $E P$ is rigid. If $\Gamma \in \mathcal{G}_{\mathfrak{p}}$, choose $\Delta \in \mathcal{B}_{\mathfrak{p}}$ with $\alpha(\Delta)=\varphi(\Gamma)$. If $\Delta \in \mathcal{B}_{\mathfrak{p}}$, choose $\Gamma \in \mathcal{G}_{\mathfrak{p}}$ with $\varphi(\Gamma)=\alpha(\Delta)$. Since $\alpha$ is rigid, $\gamma_{\Gamma}=\left.\left(\left.\alpha\right|_{\Delta}\right)^{-1} \circ \varphi\right|_{\Gamma}$ maps $\Gamma$ onto $\Delta$ and satisfies $\alpha \circ \gamma_{\Gamma}=\left.\varphi\right|_{\Gamma}$.

Proposition 4.4. Every rigid deficient embedding problem is locally solvable.

Proof. Suppose EP is rigid and deficient. By Lemma 4.3, (LS) holds for $\mathfrak{p} \in \mathfrak{S}$. Since B is deficient, so is $\mathbf{A}$, hence if $G_{0} \in \mathcal{G}_{0}$, then $\varphi\left(G_{0}\right)=1$. Thus, $(\underline{L S})$ is satisfied for $\mathfrak{p}=0$.

Definition 4.5. Let $\varphi: \mathbf{G} \rightarrow \mathbf{A}$ and $\alpha: \mathbf{B} \rightarrow \mathbf{A}$ be homomorphisms of deficient group piles. Define the (asymmetric) rigid product of $B$ and $G$ over $A$ as $\mathbf{B} \times{ }_{\mathbf{A}}^{\text {rig }} \mathbf{G}=\left(H, \mathcal{H}_{\mathfrak{p}}\right)_{\mathfrak{p} \in \mathfrak{S}}$, where $H=B \times{ }_{A} G$ is the fiber product and

$$
\mathcal{H}_{\mathfrak{p}}=\left\{\Gamma \in \operatorname{Subgr}(H): \beta(\Gamma) \in \mathcal{G}_{\mathfrak{p}}, \pi(\Gamma) \in \mathcal{B}_{\mathfrak{p}},\left.\beta\right|_{\Gamma} \text { is injective }\right\},
$$

with $\pi: H \rightarrow B$ and $\beta: H \rightarrow G$ the natural projection maps.

Lemma 4.6. Let $E P=(\varphi: \mathbf{G} \rightarrow \mathbf{A}, \alpha: \mathbf{B} \rightarrow \mathbf{A})$ be a locally solvable embedding problem of finite deficient group piles. Then the $\mathbf{B} \times{ }_{\mathbf{A}}^{\mathrm{rig}} \mathbf{G}$ is a deficient group pile, $\pi: \mathbf{B} \times{ }_{\mathbf{A}}^{\mathrm{rig}} \mathbf{G} \rightarrow \mathbf{B}$ is an epimorphism and $\beta: \mathbf{B} \times{ }_{\mathbf{A}}^{\mathrm{rig}} \mathbf{G} \rightarrow \mathbf{G}$ is a rigid epimorphism.

Proof. Since $\mathcal{G}_{\mathfrak{p}}$ is $G$-invariant and $\mathcal{B}_{\mathfrak{p}}$ is $B$-invariant, $\mathcal{H}_{\mathfrak{p}}$ is $H$-invariant. Since $H$ is finite, $\mathcal{H}_{\mathfrak{p}}$ is closed. The projections $\beta$ and $\pi$ are surjective and, by the definition of $\mathcal{H}_{\mathfrak{p}}$, homomorphisms of group piles. The definition of $\mathcal{H}_{\mathfrak{p}}$ also gives that $\beta$ is rigid. Given $G_{1} \in \mathcal{G}_{\mathfrak{p}}$, there is $B_{1} \in \mathcal{B}_{\mathfrak{p}}$ and an epimorphism $\gamma: G_{1} \rightarrow B_{1}$ with $\alpha \circ \gamma=\left.\varphi\right|_{G_{1}}$. It defines a homomorphism $\hat{\gamma}: G_{1} \rightarrow H$ with $\beta \circ \hat{\gamma}=\operatorname{id}_{G_{1}}$ and $\pi \circ \hat{\gamma}=\gamma$. Let $H_{1}=\hat{\gamma}\left(G_{1}\right)$. Then $\beta\left(H_{1}\right)=G_{1} \in \mathcal{G}_{\mathfrak{p}}$ and $\pi\left(H_{1}\right)=\gamma\left(G_{1}\right)=B_{1} \in \mathcal{B}_{\mathfrak{p}}$. Furthermore, since $\beta \circ \hat{\gamma}=\operatorname{id}_{G_{1}},\left.\beta\right|_{H_{1}}$ is injective, so $H_{1} \in \mathcal{H}_{\mathfrak{p}}$. Similarly, given $B_{1} \in \mathcal{B}_{\mathfrak{p}}$, there is $H_{1} \in \mathcal{H}_{\mathfrak{p}}$ with $\pi\left(H_{1}\right)=B_{1}$. Therefore, $\beta$ and $\pi$ are epimorphisms of group piles.

Remark 4.7. The rigid product can be seen as a canonical version of [HJP09, LemmaConstruction 4.2]. One could define it as a sub-group pile of the fiber product in the category of deficient group piles, which always exists.

Lemma 4.8. Let $(\varphi: \mathbf{G} \rightarrow \mathbf{A}, \alpha: \mathbf{B} \rightarrow \mathbf{A})$ be a locally solvable embedding problem. Then for every normal subgroup $N \triangleleft B$, the induced embedding problem $(\mathbf{G} \rightarrow \mathbf{A} / \alpha(N), \mathbf{B} / N \rightarrow$ $\mathbf{A} / \alpha(N))$ is also locally solvable.

Proof. Let $\tilde{\mathbf{B}}=\left(\tilde{B}, \tilde{\mathcal{B}}_{0}, \tilde{\mathcal{B}}_{\mathfrak{p}}\right)_{\mathfrak{p} \in \mathfrak{S}}=\mathbf{B} / N$ and $\tilde{\mathbf{A}}=\left(\tilde{A}, \tilde{\mathcal{A}}_{0}, \tilde{\mathcal{A}}_{\mathfrak{p}}\right)_{\mathfrak{p} \in \mathfrak{S}}=\mathbf{A} / \alpha(N)$, and let $\pi: \mathbf{B} \rightarrow \tilde{\mathbf{B}}, \tilde{\pi}: \mathbf{A} \rightarrow \tilde{\mathbf{A}}$ be the quotient maps and $\tilde{\alpha}: \tilde{\mathbf{B}} \rightarrow \tilde{\mathbf{A}}$ the induced epimorphism. Then $\tilde{\pi} \circ \alpha=\tilde{\alpha} \circ \pi$. We have to prove that the embedding problem $(\tilde{\pi} \circ \varphi, \tilde{\alpha})$ is locally solvable.

Let $\mathfrak{p} \in\{0\} \cup \mathfrak{S}$ and let $\Gamma \in \mathcal{G}_{\mathfrak{p}}$ be given. Then there is a $\Delta \in \mathcal{B}_{\mathfrak{p}}$ and an epimorphism $\gamma_{\Gamma}: \Gamma \rightarrow \Delta$ with $\alpha \circ \gamma_{\Gamma}=\left.\varphi\right|_{\Gamma}$. Let $\Lambda=\pi(\Delta) \in \tilde{\mathcal{B}}_{\mathfrak{p}}$. Then $\pi \circ \gamma_{\Gamma}: \Gamma \rightarrow \Lambda$ is an epimorphism with $\tilde{\alpha} \circ\left(\pi \circ \gamma_{\Gamma}\right)=\tilde{\pi} \circ \alpha \circ \gamma_{\Gamma}=\left.(\tilde{\pi} \circ \varphi)\right|_{\Gamma}$. 
Conversely, let $\Lambda \in \tilde{\mathcal{B}}_{\mathfrak{p}}$ be given. Choose $\Delta \in \mathcal{B}_{\mathfrak{p}}$ with $\pi(\Delta)=\Lambda$. Then there is a $\Gamma \in \mathcal{G}_{\mathfrak{p}}$ and an epimorphism $\gamma_{\Gamma}: \Gamma \rightarrow \Delta$ with $\alpha \circ \gamma_{\Gamma}=\left.\varphi\right|_{\Gamma}$. Hence, $\pi \circ \gamma_{\Gamma}: \Gamma \rightarrow \Lambda$ is an epimorphism with $\tilde{\alpha} \circ\left(\pi \circ \gamma_{\Gamma}\right)=\tilde{\pi} \circ \alpha \circ \gamma_{\Gamma}=\left.(\tilde{\pi} \circ \varphi)\right|_{\Gamma}$.

Lemma 4.9. Let $(\varphi: \mathbf{G} \rightarrow \mathbf{A}, \alpha: \mathbf{B} \rightarrow \mathbf{A})$ be a locally solvable finite embedding problem. Then there exists an open normal subgroup $N \triangleleft G$ with $N \leq \operatorname{Ker}(\varphi)$ such that the induced embedding problem $(\mathbf{G} / N \rightarrow \mathbf{A}, \mathbf{B} \rightarrow \mathbf{A})$ is locally solvable.

Proof. This is a special case of [HJP09, Lemma 4.1].

The following proposition is closely related to [HJP09, Lemma 4.3], which we need to reprove because we have to take $e$-boundedness into consideration.

Proposition 4.10. Let $(\varphi: \mathbf{G} \rightarrow \mathbf{A}, \alpha: \mathbf{B} \rightarrow \mathbf{A})$ be a locally solvable e-bounded finite embedding problem where $\mathbf{G}$ is e-bounded. Then it can be dominated by a rigid e-bounded finite embedding problem, i.e. there exist epimorphisms $\hat{\alpha}: \hat{\mathbf{B}} \rightarrow \hat{\mathbf{A}}, \hat{\varphi}: \mathbf{G} \rightarrow \hat{\mathbf{A}}, \hat{\beta}: \hat{\mathbf{A}} \rightarrow$ $\mathbf{A}, \beta: \hat{\mathbf{B}} \rightarrow \mathbf{B}$ such that $\varphi=\hat{\beta} \circ \hat{\varphi}$ and $\hat{\beta} \circ \hat{\alpha}=\alpha \circ \beta$, and $(\hat{\varphi}, \hat{\alpha})$ is a rigid e-bounded finite embedding problem.

Proof. By Lemma 4.9, there are a finite group pile $\hat{\mathbf{A}}$ and epimorphisms $\hat{\varphi}: \mathbf{G} \rightarrow \hat{\mathbf{A}}$, $\hat{\beta}: \hat{\mathbf{A}} \rightarrow \mathbf{A}$ with $\varphi=\hat{\beta} \circ \hat{\varphi}$ such that $(\hat{\beta}, \alpha)$ is a locally solvable embedding problem. Since $\mathbf{G}$ is $e$-bounded, also $\hat{\mathbf{A}}$ is $e$-bounded (Lemma 3.15).

Let $\tilde{\mathbf{B}}=\mathbf{B}^{\circ} \times{ }_{\mathbf{A}^{\circ}}^{\text {rig }} \hat{\mathbf{A}}^{\circ}$ be the rigid product and let $\tilde{\alpha}: \tilde{\mathbf{B}} \rightarrow \hat{\mathbf{A}}^{\circ}$ and $\tilde{\beta}: \tilde{\mathbf{B}} \rightarrow \mathbf{B}^{\circ}$ be the projections. By Lemma 4.6, $\tilde{\alpha}$ is a rigid epimorphism and $\tilde{\beta}$ is an epimorphism. Choose $\hat{A}_{0} \in \hat{\mathcal{A}}_{0}$ and $B_{0} \in \mathcal{B}_{0}$ and an epimorphism $\gamma_{0}: \hat{A}_{0} \rightarrow B_{0}$ with $\alpha \circ \gamma_{0}=\left.\hat{\beta}\right|_{\hat{A}_{0}}$. Then $\gamma_{0}$ defines a homomorphism $\hat{\gamma}_{0}: \hat{A}_{0} \rightarrow \tilde{B}$ with $\tilde{\alpha} \circ \hat{\gamma}_{0}=\operatorname{id}_{\hat{A}_{0}}$ and $\tilde{\beta} \circ \hat{\gamma}_{0}=\gamma_{0}$. Let $\tilde{B}_{0}=\hat{\gamma}_{0}\left(\hat{A}_{0}\right)$ and note that $\tilde{\alpha}\left(\tilde{B}_{0}\right)=\hat{A}_{0}$ and $\tilde{\beta}\left(\tilde{B}_{0}\right)=B_{0}$.

We have that $\operatorname{rk}\left(\tilde{B}_{0}\right) \leq \operatorname{rk}\left(\hat{A}_{0}\right) \leq e$, and since $\hat{\mathbf{A}}$ and $\mathbf{B}$ are self-generated, $\hat{A}=\left\langle\hat{A}_{0}, \hat{A}^{\prime}\right\rangle$ and $B=\left\langle B_{0}, B^{\prime}\right\rangle$. Let $\hat{B}=\left\langle\tilde{B}_{0}, \tilde{B}^{\prime}\right\rangle \leq \tilde{B}$ and $\hat{\mathcal{B}}_{0}=\left(\tilde{B}_{0}\right)^{\hat{B}}$. Then $\hat{\mathbf{B}}=\left(\hat{B}, \hat{\mathcal{B}}_{0}, \tilde{\mathcal{B}}_{\mathfrak{p}}\right)_{\mathfrak{p} \in \mathfrak{S}}$ is a self-generated group pile, and $\tilde{\alpha}(\hat{B})=\left\langle\hat{A}_{0}, \hat{A}^{\prime}\right\rangle=\hat{A}$ and $\tilde{\beta}(\hat{B})=\left\langle B_{0}, B^{\prime}\right\rangle=B$ by Lemma 3.11. Since $\hat{\mathcal{A}}_{0}=\left(\hat{A}_{0}\right)^{\hat{A}}$ and $\mathcal{B}_{0}=\left(B_{0}\right)^{B}, \tilde{\alpha}\left(\hat{\mathcal{B}}_{0}\right)=\hat{\mathcal{A}}_{0}$ and $\tilde{\beta}\left(\hat{\mathcal{B}}_{0}\right)=\mathcal{B}_{0}$, so $\left.\tilde{\alpha}\right|_{\hat{B}}$ and $\left.\tilde{\beta}\right|_{\hat{B}}$ are epimorphisms of group piles. Therefore, with $\hat{\alpha}=\left.\tilde{\alpha}\right|_{\hat{B}}$ and $\beta=\left.\tilde{\beta}\right|_{\hat{B}}$, $(\hat{\varphi}, \hat{\alpha})$ is a rigid $e$-bounded finite embedding problem which dominates $(\varphi, \alpha)$.

\section{MODEL THEORY OF GROUP PILES}

This section extends the comodel-theory of profinite groups in CDM82 (see also CDM81]) to group piles. A similar construction can be found in [Ers91. We will give full definitions but focus our proofs on the necessary extensions to the classical theory. For more on the comodel-theory of profinite groups see Cha84, Cha98, Cha02] or [Fro10].

Definition 5.1. The colanguage $\mathcal{L}_{\mathrm{co}, \mathfrak{S}}=\left\{\leq, \sqsubseteq, P,\left(G_{n}\right)_{n \in \mathbb{N}},\left(\mathcal{G}_{\mathfrak{p}, n}\right)_{\mathfrak{p} \in \mathfrak{S}, n \in \mathbb{N}}\right\}$ consists of unary relation symbols $G_{n}(n \in \mathbb{N})$, binary relation symbols $\leq$ and $\sqsubseteq$, a ternary relation symbol $P$, and $n$-ary relation symbols $\mathcal{G}_{\mathfrak{p}, n}(\mathfrak{p} \in \mathfrak{S}, n \in \mathbb{N})$.

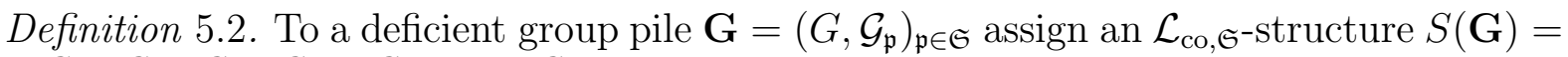
$\left(S^{\mathbf{G}}, \leq^{\mathbf{G}}, \sqsubseteq^{\mathbf{G}}, P^{\mathbf{G}},\left(G_{n}^{\mathbf{G}}\right)_{n \in \mathbb{N}},\left(\mathcal{G}_{\mathfrak{p}, n}^{\mathbf{G}}\right)_{\mathfrak{p} \in \mathfrak{S}, n \in \mathbb{N}}\right)$ as follows:

1. $S^{\mathbf{G}}=\bigcup_{N} G / N$, where $N$ runs over all open normal subgroups of $G$.

2. $x_{1} N_{1} \leq \mathbf{G} x_{2} N_{2}$ if and only if $N_{1} \subseteq N_{2}$.

3. $x_{1} N_{1} \sqsubseteq \mathbf{G} x_{2} N_{2}$ if and only if $x_{1} N_{1} \subseteq x_{2} N_{2}$.

4. $\left(x_{1} N_{1}, x_{2} N_{2}, x_{3} N_{3}\right) \in P^{\mathbf{G}}$ if and only if $N_{1}=N_{2}=N_{3}$ and $x_{1} x_{2} N_{1}=x_{3} N_{1}$.

5. $x N \in G_{n}^{\mathbf{G}}$ if and only if $(G: N) \leq n$. 
6. $\left(x_{1} N_{1}, \ldots, x_{n} N_{n}\right) \in \mathcal{G}_{\mathfrak{p}, n}^{\mathbf{G}}$ if and only if $N_{1}=\cdots=N_{n} \in G_{n}^{\mathbf{G}}$ and there is $\Gamma \in \mathcal{G}_{\mathfrak{p}}$ such that $\Gamma N_{1} / N_{1}=\left\{x_{1} N_{1}, \ldots, x_{n} N_{n}\right\}$.

Definition 5.3. An $\mathcal{L}_{\mathrm{co}, \mathfrak{S}^{-}}$structure $\mathbf{S}=\left(S, \leq, \sqsubseteq, P,\left(G_{n}\right)_{n \in \mathbb{N}},\left(\mathcal{G}_{\mathfrak{p}, n}\right)_{\mathfrak{p} \in \mathfrak{S}, n \in \mathbb{N}}\right)$ is an inverse system (of group piles) if the following statements hold:

$(1) \leq$ is a preorder with a unique largest element.

(2) If $\sim$ denotes the equivalence relation defined by $\leq$, and $[x]$ denotes the equivalence class of $x \in S$ with respect to $\sim$, then the induced partial order on $\{[x]: x \in$ $S\}=S / \sim$ is directed downwards.

(3) $G_{n}=\{x \in S:|[x]| \leq n\}$

(4) $(x, y, z) \in P$ implies $[x]=[y]=[z]$, and for each $x \in S, P \cap[x]^{3}$ is the graph of a binary operation making $[x]$ into a group.

(5) If $\left(x_{1}, \ldots, x_{n}\right) \in \mathcal{G}_{\mathfrak{p}, n}$, then $x_{1}, \ldots, x_{n} \in G_{n}$ and $\left[x_{1}\right]=\cdots=\left[x_{n}\right]$. If moreover $y_{1}, \ldots, y_{n} \in G_{n}$ and $\left\{y_{1}, \ldots, y_{n}\right\}=\left\{x_{1}, \ldots, x_{n}\right\}$, then $\left(y_{1}, \ldots, y_{n}\right) \in \mathcal{G}_{\mathfrak{p}, n}$. If $x \in G_{n}$, then, with $\mathcal{G}_{\mathfrak{p}, x}=\left\{\left\{x_{1}, \ldots, x_{n}\right\} \subseteq[x]:\left(x_{1}, \ldots, x_{n}\right) \in \mathcal{G}_{\mathfrak{p}, n}\right\}, \llbracket x \rrbracket=$ $\left([x], \mathcal{G}_{\mathfrak{p}, x}\right)_{\mathfrak{p} \in \mathfrak{S}}$ is a (finite) deficient group pile.

(6) $x \sqsubseteq y$ implies $x \leq y$, and for each $x, y \in S$ with $x \leq y$, defines an epimorphism of group piles $\pi_{x, y}$ from $\llbracket x \rrbracket$ to $\llbracket y \rrbracket$, depending only on $[x]$ and $[y]$.

(7) For all $x \leq y \leq z, \pi_{x, x}=\mathrm{id}_{\llbracket x \rrbracket}$ and $\pi_{x, z}=\pi_{y, z} \circ \pi_{x, y}$.

(8) If $N$ is a normal subgroup of $\llbracket x \rrbracket$, then there is a unique $[y]$ such that $x \leq y$ and $N=\operatorname{ker}\left(\pi_{x, y}\right)$.

(9) $S=\bigcup_{n \in \mathbb{N}} G_{n}$.

Remark 5.4. Note that for $\mathfrak{S}=\emptyset$, (15) is vacant, and the remaining axioms are exactly

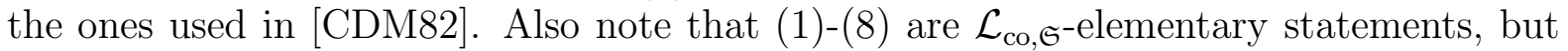
(9) is not.

Lemma 5.5. If $\mathbf{G}$ is a deficient group pile, then $S(\mathbf{G})$ is an inverse system.

Proof. This can be checked directly from the definitions.

Definition 5.6. If $\varphi: \mathbf{G} \rightarrow \mathbf{H}$ is an epimorphism of deficient group piles, $\mathbf{G}=\left(G, \mathcal{G}_{\mathfrak{p}}\right)_{\mathfrak{p} \in \mathfrak{S}}$, $\mathbf{H}=\left(H, \mathcal{H}_{\mathfrak{p}}\right)_{\mathfrak{p} \in \mathfrak{S}}$, define a map $\varphi^{*}$ of $S(\mathbf{H})$ into $S(\mathbf{G})$ by

$$
\varphi^{*}(h N)=g \varphi^{-1}(N) \in G / \varphi^{-1}(N),
$$

where $N \triangleleft H$ is open, $h \in H$, and $g \in G$ satisfies $\varphi(g)=h$.

Lemma 5.7. The map $\varphi^{*}$ is an embedding of $\mathcal{L}_{\mathrm{co}, \mathfrak{S}^{-} \text {-structures. }}$

Proof. First of all note that since $\varphi$ is a surjective homomorphism, $\varphi^{*}$ is injective and preserves the relations $\leq, \sqsubseteq P$ and $\left(G_{n}\right)_{n \in \mathbb{N}}$.

Let $h_{1} N_{1}, \ldots, h_{n} N_{n} \in S^{\mathbf{H}}$ and $\mathfrak{p} \in \mathfrak{S}$. Then $\left(h_{1} N_{1}, \ldots, h_{n} N_{n}\right) \in \mathcal{G}_{\mathfrak{p}, n}^{\mathbf{H}}$ if and only if $N_{1}=\cdots=N_{n} \in G_{n}^{\mathbf{H}}$ and there is $\Delta \in \mathcal{H}_{\mathfrak{p}}$ such that $\Delta N_{1} / N_{1}=\left\{h_{1} N_{1}, \ldots, h_{n} N_{n}\right\}$. Since $\varphi\left(\mathcal{G}_{\mathfrak{p}}\right)=\mathcal{H}_{\mathfrak{p}}$, this is the case if and only if $\varphi^{*}\left(N_{1}\right)=\cdots=\varphi^{*}\left(N_{n}\right) \in G_{n}^{\mathbf{G}}$ and there is $\Gamma \in \mathcal{G}_{\mathfrak{p}}$ such that $\Gamma \varphi^{-1}\left(N_{1}\right) / \varphi^{-1}\left(N_{1}\right)=\left\{\varphi^{*}\left(h_{1} N_{1}\right), \ldots, \varphi^{*}\left(h_{n} N_{n}\right)\right\}$. This is equivalent to $\left(\varphi^{*}\left(h_{1} N_{1}\right), \ldots, \varphi^{*}\left(h_{n} N_{n}\right)\right) \in \mathcal{G}_{\mathfrak{p}, n}^{\mathbf{G}}$, so $\varphi^{*}$ also preserves the relations $\left(\mathcal{G}_{\mathfrak{p}, n}\right)_{\mathfrak{p} \in \mathfrak{S}, n \in \mathbb{N}}$.

Definition 5.8. We assign to each inverse system $\mathbf{S}=\left(S, \leq, \sqsubseteq, P,\left(G_{n}\right)_{n \in \mathbb{N}},\left(\mathcal{G}_{\mathfrak{p}, n}\right)_{\mathfrak{p} \in \mathfrak{S}, n \in \mathbb{N}}\right)$ a deficient group pile $G(\mathbf{S})$ as follows: By axioms (1), (2) and (4) the $[x]$ are a family of groups, which by (3) and (9) are all finite. By (5), the $\llbracket x \rrbracket$ are finite groups piles. By axioms (6) and (7), the maps $\pi_{x, y}$ turns these group piles into an inverse system in the category of group piles with epimorphisms. Let $G(\mathbf{S})=\left(G^{\mathbf{S}}, \mathcal{G}_{\mathfrak{p}}^{\mathbf{S}}\right)_{\mathfrak{p} \in \mathfrak{S}}:=\lim _{\leftarrow \in S / \sim} \llbracket x \rrbracket$ be the inverse limit, cf. Lemma 3.8. 
Lemma 5.9. The maps $S$ and $G$ are quasi-inverse to each other.

Proof. Let $\mathbf{G}=\left(G, \mathcal{G}_{\mathfrak{p}}\right)_{\mathfrak{p} \in \mathfrak{S}}$ be a deficient group pile. Since $\mathcal{G}_{\mathfrak{p}}$ is closed, $\mathcal{G}_{\mathfrak{p}}=\lim _{N} \mathcal{G}_{\mathfrak{p}, N}$, where $N$ runs over all open normal subgroups of $G$. Therefore $G(S(\mathbf{G})) \cong \mathbf{G}$.

Conversely, let $\mathbf{S}$ be an inverse system. Given $x \in S$, let $N_{x}$ be the kernel of the natural projection $G^{\mathbf{S}} \rightarrow \llbracket x \rrbracket$. Define $\psi: \mathbf{S} \rightarrow S(G(\mathbf{S}))$ to send $x$ to its image under the natural isomorphism $\llbracket x \rrbracket \cong G^{\mathbf{S}} / N_{x} \subseteq S^{G(\mathbf{S})}$. Then $\psi$ is injective and preserves the relations $\leq, \sqsubseteq P, G_{n}$ and $\mathcal{G}_{\mathfrak{p}, n}$. By axiom (8), $\psi$ is surjective. Thus $\psi$ gives an isomorphism $\mathbf{S} \cong S(G(\mathbf{S}))$.

Remark 5.10. Given the preceding lemma, we will sometimes identify an inverse system $\mathbf{S}$ with $S(G(\mathbf{S}))$. In particular, we will treat elements of $\mathbf{S}$ as cosets $x N$ of open normal subgroups of the group pile $G(\mathbf{S})$.

Definition 5.11. If $\psi: \mathbf{T} \rightarrow \mathbf{S}$ is an embedding of inverse systems, define an epimorphism of profinite groups $\psi^{*}: G^{\mathbf{S}} \rightarrow G^{\mathbf{T}}$ as follows:

Since $\psi$ preserves the relation $\leq$, it also preserves the relation $\sim$, i.e. $\psi([x]) \leq[\psi(x)]$. Since $\psi$ preserves the relations $G_{n}(n \in \mathbb{N}), \psi$ gives a bijection $[x] \rightarrow[\psi(x)]$. Since $\psi$ preserves the relation $P$, this bijection is an isomorphism of groups. Since $\psi$ preserves the relation $\sqsubseteq$, the inverse system of finite groups $([\psi(x)])_{x \in T / \sim}$ with homomorphisms $\pi_{\psi(x), \psi(y)}$ is isomorphic to the inverse system of finite groups $([x])_{x \in T / \sim}$ with homomorphisms $\pi_{x, y}$. This gives a natural epimorphism

$$
\psi^{*}: G^{\mathbf{S}}=\lim _{y \in S / \sim}[x] \rightarrow \lim _{x \in T / \sim}[\psi(x)] \cong \lim _{x \in T / \sim}[x]=G^{\mathbf{T}} .
$$

Lemma 5.12. The map $\psi^{*}$ extends to an epimorphism $\psi^{*}: G(\mathbf{S}) \rightarrow G(\mathbf{T})$ of group piles.

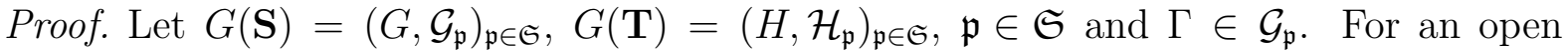
normal subgroup $M$ of $H$ one may check that $\psi\left(\psi^{*}(\Gamma) M / M\right)=\Gamma N / N \in \mathcal{G}_{\mathfrak{p}, N}$, where $\psi(H / M)=G / N$. Since $\psi$ preserves the relations $\mathcal{G}_{\mathfrak{p}, n}$, this implies $\psi^{*}(\Gamma) M / M \in \mathcal{H}_{\mathfrak{p}, M}$. So $\psi^{*}(\Gamma) \in \lim _{N} \mathcal{H}_{\mathfrak{p}, N}=\mathcal{H}_{\mathfrak{p}}$.

Conversely, let $\Gamma \in \mathcal{H}_{\mathfrak{p}}$ be given. The family $\psi(\Gamma M / M) \in \mathcal{G}_{\mathfrak{p}, N}, M \triangleleft H$ open and $\psi(H / M)=G / N$, is compatible with respect to the $\pi_{x, y}$, hence there exists $\Delta \in \mathcal{G}_{\mathfrak{p}}$ with $\psi(\Gamma M / M)=\Delta N / N$ for all $M \triangleleft H$ open, where $\psi(H / M)=G / N$, and thus $\psi^{*}(\Delta)=\Gamma$. Thus $\psi^{*}\left(\mathcal{G}_{\mathfrak{p}}\right)=\mathcal{H}_{\mathfrak{p}}$ for all $\mathfrak{p} \in \mathfrak{S}$, so $\psi^{*}$ is an epimorphism of group piles.

Proposition 5.13. The map $S$ defines an equivalence of categories between the category of deficient group piles (with epimorphisms) and the category of inverse system (with embeddings), with quasi-inverse $G$.

Proof. Note that $S$ and $G$ are in fact functorial. Now apply the previous lemmas.

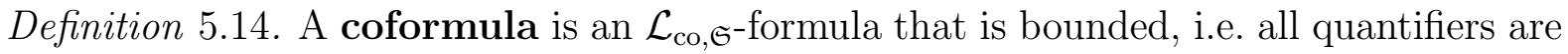
of the form $\left(\exists v \in G_{n}\right)$. A cosentence is a coformula without free variables.

A group pile $\mathbf{G}$ cosatisfies a set $\Sigma$ of coformulas with free variables $V$ (or is a comodel of $\Sigma)$ if there are elements $x_{v} \in S(\mathbf{G}), v \in V$, such that $S(\mathbf{G}) \models \varphi\left(x_{v}, v \in V\right)$ for all $\varphi \in \Sigma$. A cotheory $T$ is a set of cosentences.

The cocardinality of a group pile $\mathbf{G}$ is the cardinality of $S(\mathbf{G})$. A set $\Sigma$ of coformulas with parameters in some inverse system $\mathbf{S}$ is ranked if for every variable $v$ that occurs in some formula $\varphi \in \Sigma$, also $G_{n}(v) \in \Sigma$ for some $n \in \mathbb{N}$. A group pile $\mathbf{G}$ is $\kappa$-cosaturated if every ranked set $\Sigma$ of coformulas with parameters in $S(\mathbf{G})$ with $|\Sigma|<\kappa$ is cosatisfied in $\mathbf{G}$, provided that every finite subset of $\Sigma$ is cosatisfied in $\mathbf{G}$. 
Remark 5.15. An inverse system $\mathbf{S}$ in the language $\mathcal{L}_{\mathrm{co}, \mathfrak{S}}$ can also be viewed as an $\omega$-sorted structure $\mathbf{S}^{\omega}$ in a language $\mathcal{L}_{\mathrm{co}, \mathfrak{S}}^{\omega}$, where the $n$-th sort consists of the $s \in G_{n} \backslash G_{n-1}$, and for each $k$-ary relation relation $R$ in $\mathcal{L}_{\mathrm{co}, \mathfrak{S}}$ we have an $\omega^{k}$-family of $k$-ary relations $R^{n_{1}, \ldots, n_{k}}$ in $\mathcal{L}_{\mathrm{co}, \mathfrak{S}}^{\omega}$, cf. Cha98, §1]. Clearly, every $\mathcal{L}_{\mathrm{co}, \mathfrak{S}}$-formula can be translated to a corresponding $\mathcal{L}_{\mathrm{co}, \mathfrak{S}^{-}}^{\omega}$ formula, and vice versa.

\section{6. e-FREE C-PILES}

In this section, we generalize the Cantor group piles of [HJP09].

Definition 6.1. An $e$-free $\mathbf{C}$-pile is an $e$-generated deficient group pile $\mathbf{G}$ for which every rigid $e$-generated deficient finite embedding problem is solvable (cf. Def. 3.9).

Lemma 6.2. If $\mathbf{G}$ is an e-free $C$-pile, then $\bar{G} \cong \hat{F}_{e}$.

Proof. If $B$ is a finite group with $\operatorname{rk}(B) \leq e$, then $(\mathbf{G} \rightarrow 1, B \rightarrow 1)$ is a rigid $e$-generated deficient finite embedding problem for $\mathbf{G}$, so it has a solution by assumption. Therefore, by Lemma 3.13, every finite group $B$ with $\operatorname{rk}(B) \leq e$ is a quotient of $\bar{G}$. Since $\operatorname{rk}(\bar{G}) \leq e$, this implies that $\bar{G} \cong \hat{F}_{e}$, cf. [FJ08, 16.10.7].

Lemma 6.3. Let $\mathbf{G}$ be an e-bounded group pile, and let $\left(\varphi: \mathbf{G}^{\circ} \rightarrow \tilde{\mathbf{A}}, \alpha: \tilde{\mathbf{B}} \rightarrow \tilde{\mathbf{A}}\right)$ be a locally solvable e-generated deficient finite embedding problem. If $G_{0} \cong \hat{F}_{e}$ for $G_{0} \in \mathcal{G}_{0}$, then there exist $\mathbf{A}$ and $\mathbf{B}$ with $\mathbf{A}^{\circ}=\tilde{\mathbf{A}}$ and $\mathbf{B}^{\circ}=\tilde{\mathbf{B}}$ such that $(\varphi: \mathbf{G} \rightarrow \mathbf{A}, \alpha: \mathbf{B} \rightarrow \mathbf{A})$ is a locally solvable e-bounded finite embedding problem.

Proof. Let $G_{0} \in \mathcal{G}_{0}$ and $A_{0}=\varphi\left(G_{0}\right)$. Then $G=\left\langle G_{0}, G^{\prime}\right\rangle$ implies $\tilde{A}=\left\langle A_{0}, \tilde{A}^{\prime}\right\rangle$ (Lemma 3.11), so $\mathbf{A}=\left(\tilde{A},\left(A_{0}\right)^{\tilde{A}}, \tilde{\mathcal{A}}_{\mathfrak{p}}\right)_{\mathfrak{p} \in \mathfrak{S}}$ is $e$-bounded. By Lemma 3.17, there exists an $e$-bounded group pile $\mathbf{B}=\left(\tilde{B},\left(B_{0}\right)^{\tilde{B}}, \tilde{\mathcal{B}}_{\mathfrak{p}}\right)_{\mathfrak{p} \in \mathfrak{S}}$ such that $\alpha: \mathbf{B} \rightarrow \mathbf{A}$ is an epimorphism. Without loss of generality assume that $\alpha\left(B_{0}\right)=A_{0}$. We claim that $E P=(\varphi: \mathbf{G} \rightarrow \mathbf{A}, \alpha: \mathbf{B} \rightarrow \mathbf{A})$ is locally solvable. Clearly it satisfies $(\underline{L S})$ for $\mathfrak{p} \in \mathfrak{S}$. Since $G_{0} \cong \hat{F}_{e}$ and $\mathbf{B}$ is $e$-bounded, there exists an epimorphism $\gamma_{0}: G_{0} \rightarrow B_{0}$ with $\alpha \circ \gamma_{0}=\left.\varphi\right|_{G_{0}}$, cf. [FJ08, 17.7.3]. Thus, by Lemma 4.2, $E P$ satisfies $(\underline{L S})$ for $\mathfrak{p}=0$.

Proposition 6.4. Every locally solvable e-generated deficient finite embedding problem for an e-free C-pile $\mathbf{G}$ is solvable.

Proof. Let $E P=(\varphi: \mathbf{G} \rightarrow \tilde{\mathbf{A}}, \alpha: \tilde{\mathbf{B}} \rightarrow \tilde{\mathbf{A}})$ be a locally solvable $e$-generated deficient finite embedding problem for G. By Lemma 6.2, $\bar{G} \cong \hat{F}_{e}$. Let $G_{0} \leq G$ be a subgroup of rank at most $e$ that under the quotient map $G \rightarrow \bar{G}$ maps onto $\bar{G} \cong \hat{F}_{e}$. Since every finite group generated by $e$ elements is a quotient of $\hat{F}_{e}$, it is also a quotient of $G_{0}$, and thus $G_{0} \cong \hat{F}_{e}$, cf. [FJ08, 16.10.7]. Moreover, $\mathbf{G}^{*}=\left(G,\left(G_{0}\right)^{G}, \mathcal{G}_{\mathfrak{p}}\right)_{\mathfrak{p} \in \mathfrak{S}}$ is $e$-bounded. By Lemma 6.3, there exist $\mathbf{A}$ and $\mathbf{B}$ with $\mathbf{A}^{\circ}=\tilde{\mathbf{A}}$ and $\mathbf{B}^{\circ}=\tilde{\mathbf{B}}$ such that $E P_{1}=\left(\varphi: \mathbf{G}^{*} \rightarrow \mathbf{A}, \alpha: \mathbf{B} \rightarrow \mathbf{A}\right)$ is locally solvable and $e$-bounded. By Proposition 4.10, $E P_{1}$ can be dominated by a rigid $e$-bounded finite embedding problem $E P_{2}$. The deficient reduct of $E P_{2}$ is a rigid $e$ generated deficient finite embedding problem, hence has a solution. It induces a solution of $E P$.

Example 6.5. For each $\mathfrak{p} \in \mathfrak{S}$, let $\Gamma_{\mathfrak{p}}$ be a profinite group and $T_{\mathfrak{p}}$ a profinite space, and let $\Gamma_{0}=\hat{F}_{e}$ be the free profinite group of rank e. Then [HJP09, §5] constructs from this data a certain group pile $\mathbf{G}_{T}$, which we call the e-free semi-constant group pile of

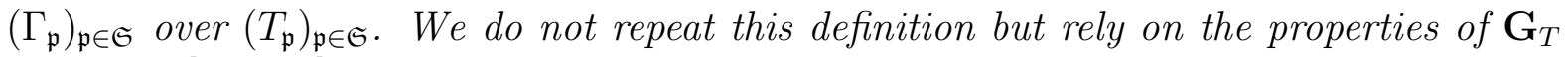
proven in [HJP09]. 
Lemma 6.6. The e-free semi-constant group pile $\mathbf{G}$ of $\left(\Gamma_{\mathfrak{p}}\right)_{\mathfrak{p} \in \mathfrak{S}}$ over $\left(T_{\mathfrak{p}}\right)_{\mathfrak{p} \in \mathfrak{S}}$ is an ebounded self-generated group pile.

Proof. By [HJP09, Proposition 5.3(c)], G is self-generated. By the construction, every $G_{0} \in \mathcal{G}_{0}$ is isomorphic to $\Gamma_{0}=\hat{F}_{e}$, hence $\mathbf{G}$ is $e$-bounded.

Proposition 6.7. Let $\mathbf{G}$ be an e-free semi-constant group pile of non-trivial profinite

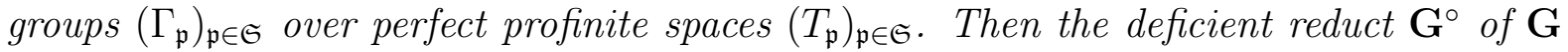
is an e-free C-pile.

Proof. By Lemma 6.6, $\mathbf{G}$ is e-bounded, so $\mathbf{G}^{\circ}$ is $e$-generated. Let $E P=\left(\varphi: \mathbf{G}^{\circ} \rightarrow\right.$ $\tilde{\mathbf{A}}, \alpha: \tilde{\mathbf{B}} \rightarrow \tilde{\mathbf{A}}$ ) be a rigid $e$-generated deficient finite embedding problem for $\mathbf{G}^{\circ}$. By Lemma 4.4, $E P$ is locally solvable. By Lemma 6.3 there exist $\mathbf{A}$ and $\mathbf{B}$ with $\mathbf{A}^{\circ}=\tilde{\mathbf{A}}$ and $\mathbf{B}^{\circ}=\tilde{\mathbf{B}}$ such that $(\varphi: \mathbf{G} \rightarrow \mathbf{A}, \alpha: \mathbf{B} \rightarrow \mathbf{A})$ is a locally solvable $e$-bounded (and hence self-generated) embedding problem. By [HJP09, Proposition 5.3(h)], this embedding problem has a solution, which in turn induces a solution of $E P$.

Definition 6.8. Let the cotheory $T_{\mathrm{C}, \mathfrak{S}, e}^{\mathrm{co}}$ consist of:

(1) For $n \in \mathbb{N}$ a cosentence about a group pile $\mathbf{G}=\left(G, \mathcal{G}_{\mathfrak{p}}\right)_{\mathfrak{p} \in \mathfrak{S}}$ stating that for each $N \triangleleft G$ with $(G: N) \leq n$, the finite quotient $\mathbf{G} / N$ is $e$-generated.

(2) For $n, k \in \mathbb{N}$ a cosentence about a group pile $\mathbf{G}=\left(G, \mathcal{G}_{\mathfrak{p}}\right)_{\mathfrak{p} \in \mathfrak{S}}$ stating that for every $N \triangleleft G$ with $(G: N) \leq n$ and every rigid epimorphism $\alpha: \mathbf{B} \rightarrow \mathbf{G} / N$ with $\mathbf{B}$ an $e$-generated deficient group pile of order $k$, there is an $M \triangleleft G$ with $(G: M) \leq k$ and $M \leq N$ and an isomorphism $\beta: \mathbf{G} / M \rightarrow \mathbf{B}$ such that $\alpha \circ \beta$ is the natural $\operatorname{map} \mathbf{G} / M \rightarrow \mathbf{G} / N$.

Proposition 6.9. A deficient group pile $\mathbf{G}$ is an e-free $C$-pile if and only if it cosatisfies $T_{\mathrm{C}, \mathfrak{S}, e}^{\mathrm{co}}$.

Proof. By Proposition 3.16, G cosatisfies (1) if and only if $\mathbf{G}$ is e-generated. And (2) just says that all rigid $e$-generated deficient finite embedding problems for $\mathbf{G}$ are solvable.

Proposition 6.10. Let $\mathbf{G}$ be an $\aleph_{1}$-cosaturated e-free $C$-pile. Then every rigid e-generated deficient embedding problem $(\varphi: \mathbf{G} \rightarrow \mathbf{A}, \alpha: \mathbf{B} \rightarrow \mathbf{A})$ with $\operatorname{rk}(\mathbf{B}) \leq \aleph_{0}$ is solvable.

Proof. Since $\operatorname{rk}(B) \leq \aleph_{0}$, there is a descending sequence of open normal subgroups $N_{i} \triangleleft B$, $i \in \mathbb{N}$, with $\bigcap_{i \in \mathbb{N}} N_{i}=1$, cf. [FJ08, 17.1.7(a)]. For each $i \in \mathbb{N}$ let $\alpha_{i}: \mathbf{B} / N_{i} \rightarrow \mathbf{A} / \alpha\left(N_{i}\right)$ be the epimorphism induced by $\alpha$, and for $i \leq j \in \mathbb{N}$ let $\pi_{i}: \mathbf{A} \rightarrow \mathbf{A} / \alpha\left(N_{i}\right), \rho_{i}: \mathbf{B} \rightarrow \mathbf{B} / N_{i}$, $\rho_{j i}: \mathbf{B} / N_{j} \rightarrow \mathbf{B} / N_{i}$ be the quotient maps. Then $\alpha_{i} \circ \rho_{i}=\pi_{i} \circ \alpha$.

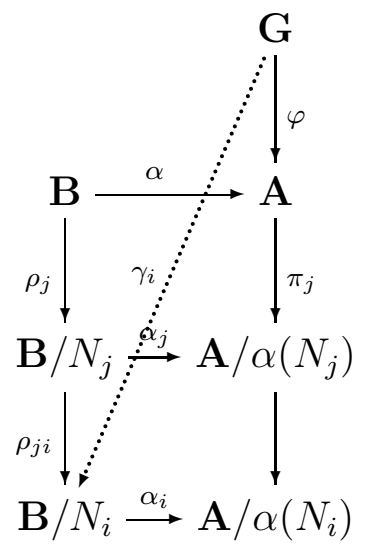

By Lemma 4.4, the rigid deficient embedding problem $(\varphi, \alpha)$ is locally solvable, hence the induced embedding problem $\left(\pi_{i} \circ \varphi, \alpha_{i}\right)$ is locally solvable by Lemma 4.8. Since $\mathbf{B}$ is 
$e$-generated, $\mathbf{B} / N_{i}$ is $e$-generated by Lemma 3.15. Hence, $\left(\pi_{i} \circ \varphi, \alpha_{i}\right)$ is a locally solvable $e$-generated deficient finite embedding problem for $\mathbf{G}$. Since $\mathbf{G}$ is an $e$-free $\mathbf{C}$-pile, this embedding problem has a solution $\gamma_{i}: \mathbf{G} \rightarrow \mathbf{B} / N_{i}$ by Proposition 6.4.

For each $i$, fix an enumeration $\mathbf{B} / N_{i}=\left\{b_{i, 1}, \ldots, b_{i, n_{i}}\right\}$ and let $a_{i, \nu}=\alpha_{i}\left(b_{i, \nu}\right) \in$ $\mathbf{A} / \alpha\left(N_{i}\right) \subseteq S(\mathbf{A})$. View $S(\mathbf{A})$ as a subset of $S(\mathbf{G})$ via $\varphi^{*}$ and let $\Sigma$ be the follow-

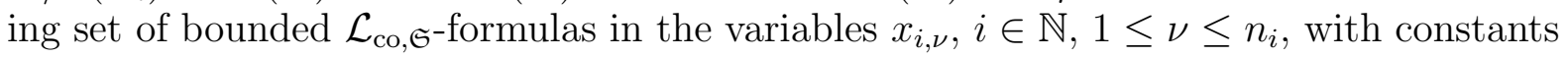
from $S(\mathbf{A})$ :

(1) For each $i$ and each $1 \leq \nu \leq n_{i}$ the $\mathcal{L}_{\mathrm{co}, \mathfrak{S}}$-formula $G_{n_{i}}\left(x_{i, \nu}\right)$.

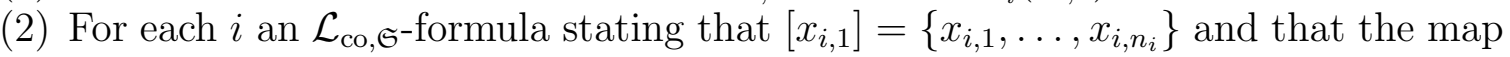
$x_{i, \nu} \mapsto b_{i, \nu}$ is an isomorphism of group piles $\beta_{i}: \llbracket x_{i, 1} \rrbracket \rightarrow \mathbf{B} / N_{i}$.

(3) For each $i$ an $\mathcal{L}_{\mathrm{co}, \mathfrak{S}}$-formula with constants from $S(\mathbf{A})$ stating that $x_{i, 1} \leq a_{i, 1}$ and $\pi_{x_{i, 1}, a_{i, 1}}\left(x_{i, \nu}\right)=a_{i, \nu}$ for all $1 \leq \nu \leq n_{i}$.

(4) For each $i \leq j$, an $\mathcal{L}_{\mathrm{co}, \mathfrak{S}}$-formula stating that $x_{j, 1} \leq x_{i, 1}$ and $\beta_{i} \circ \pi_{x_{j, 1} x_{i, 1}}=\rho_{j i} \circ \beta_{j}$.

Every finite subset $\Sigma_{0}$ of $\Sigma$ is cosatisfied in G: Let $j$ be the maximal index of a variable $x_{j, \nu}$ appearing in $\Sigma$ and for $i \leq j, 1 \leq \nu \leq n_{i}$ let $g_{i, \nu}=\gamma_{j}^{*}\left(\rho_{j i}^{*}\left(b_{i, \nu}\right)\right)$. Then $\left(g_{i, \nu}\right)_{1 \leq i \leq j, 1 \leq \nu \leq n_{i}}$ satisfies $\Sigma_{0}$. By (1), $\Sigma$ is ranked.

Thus, since $\mathbf{G}$ is $\aleph_{1}$-cosaturated, there are $\left(\tilde{g}_{i, \nu}\right)_{1 \leq i, 1 \leq \nu \leq n_{i}}$ in $\mathbf{G}$ that satisfy $\Sigma$. For each $i$, the map $b_{i, \nu} \mapsto \tilde{g}_{i, \nu}$ gives an isomorphism $\mathbf{B} / N_{i} \rightarrow \llbracket \tilde{g}_{i, 1} \rrbracket$ by (2), hence has as dual an epimorphism $\tilde{\gamma}_{i}: \mathbf{G} \rightarrow \mathbf{B} / N_{i}$, which satisfies $\alpha_{i} \circ \tilde{\gamma}_{i}=\pi_{i} \circ \varphi$ by (3). By (4), these epimorphisms are compatible, giving rise to an epimorphism $\tilde{\gamma}=\lim _{i} \tilde{\gamma}_{i}: \mathbf{G} \rightarrow$ $\lim _{i} \mathbf{B} / N_{i}=\mathbf{B}$, which then satisfies $\alpha \circ \tilde{\gamma}=\varphi$.

\section{MOdel THEORY OF PSCC FIELDS}

In the next section we will let the finite set $\mathfrak{S}$ be a set of primes and associate to each field $F$ a group pile $\operatorname{Gal}_{\mathfrak{S}}(F)$, which extends the absolute Galois group $\operatorname{Gal}(F)$ with $\mathfrak{S}$-local data. The notion of prime and the corresponding local-global principle PSCC we use is the one developed in Feh13. We now briefly recall the main definitions and results but refer to [Feh13, §2-3] for further detail.1. Basics on real closed and $p$-adically closed fields are summarized in Appendix $\mathrm{A}$ and $\mathrm{B}$.

Definition 7.1. For a field $F$ of characteristic zero we denote by $\tilde{F}$ a fixed algebraic closure of $F$, and by $\operatorname{Gal}(F)=\operatorname{Gal}(\tilde{F} / F)$ the absolute Galois group of $F$.

Definition 7.2. A prime 2 of a field $K$ is either an ordering of $K$ or an equivalence class of $p$-valuations on $K$, for some prime number $p$. It is local if the ordering is archimedean resp. if the value group is isomorphic to $\mathbb{Z}$. If $\mathfrak{P}$ is a prime of $K$, we denote by $\mathrm{CC}(K, \mathfrak{P})$ the set of all real resp. $p$-adic closures of $(K, \mathfrak{P})$ inside $\tilde{K}$. If $\mathfrak{p}$ is a prime of $K$ and $F / K$ is a field extension, we denote by $\mathcal{S}_{\mathfrak{p}}(F)$ the set of all primes $\mathfrak{P}$ of $F$ that lie above $\mathfrak{p}$ (we write this as $\left.\mathfrak{P}\right|_{K}=\mathfrak{p}$ ) and are of the same type, and by $\mathrm{CC}_{\mathfrak{p}}(F)$ the union of all $\mathrm{CC}(F, \mathfrak{P}), \mathfrak{P} \in \mathcal{S}_{\mathfrak{p}}(F)$, cf. [Feh13, Def. 3.4, Def. 3.7, Def. 4.2].

Setting 7.3. For the rest of this work let $\mathfrak{S}$ be a finite set of local primes of a field $K$ of characteristic zero, and let $F / K$ be a field extension. For $\mathfrak{p} \in \mathfrak{S}$ fix a closure $K_{\mathfrak{p}} \in \mathrm{CC}_{\mathfrak{p}}(K)$.

\footnotetext{
${ }^{1}$ The reader who want to check these details should be aware of the fact that, in the notation of [Feh13, here we consider only the case of relative type $\tau=(1,1)$, so for example the PSCC property is there called $\mathrm{P} S^{\tau} \mathrm{CC}$ with $S=\mathfrak{S}$ and $\tau=(1,1)$.

${ }^{2}$ called a classical prime in [Feh13]
} 
Lemma 7.4. Let $K \subseteq E \subseteq F, \mathfrak{p} \in \mathfrak{S}, \mathfrak{Q} \in \mathcal{S}_{\mathfrak{p}}(F)$ and $\mathfrak{P}=\left.\mathfrak{Q}\right|_{E} \in \mathcal{S}_{\mathfrak{p}}(E)$. If $F^{\prime} \in$ $\mathrm{CC}(F, \mathfrak{Q})$, then $E^{\prime}:=F^{\prime} \cap \tilde{E} \in \mathrm{CC}(E, \mathfrak{P})$ and res: $\operatorname{Gal}\left(F^{\prime}\right) \rightarrow \operatorname{Gal}\left(E^{\prime}\right)$ is an isomorphism. In particular, $F^{\prime} \cap \tilde{E} \in \mathrm{CC}_{\mathfrak{p}}(E)$ for any $F^{\prime} \in \mathrm{CC}_{\mathfrak{p}}(F)$.

Proof. The field $E^{\prime}$ is algebraically closed in the real closed resp. $p$-adically closed field $F^{\prime}$, so it is real closed resp. $p$-adically closed itself, see Lemma A.2 and Lemma B.1, Let $\mathfrak{Q}^{\prime}$ be the unique prime of $F^{\prime}$ over $\mathfrak{Q}$. Then $\mathfrak{P}^{\prime}=\left.\mathfrak{Q}^{\prime}\right|_{E^{\prime}}$ is the unique prime of $E^{\prime}$ of the same type as $\mathfrak{p}$, so $E^{\prime} \in \mathrm{CC}\left(E,\left.\mathfrak{P}^{\prime}\right|_{E}\right)$. Since $\left.\mathfrak{P}^{\prime}\right|_{E}=\left.\mathfrak{Q}^{\prime}\right|_{E}=\mathfrak{P}$, it follows that $E^{\prime} \in \mathrm{CC}(E, \mathfrak{P})$.

Since $E^{\prime} \equiv F^{\prime}$ by model completeness (Proposition A.5 and Proposition B.3), and $\operatorname{Gal}\left(F^{\prime}\right)$ is finitely generated (Proposition A.3 and Proposition B.5), $\operatorname{Gal}\left(E^{\prime}\right) \cong \operatorname{Gal}\left(F^{\prime}\right)$ by [FJ08, 20.4.6]. Thus the epimorphism res: $\operatorname{Gal}\left(F^{\prime}\right) \rightarrow \operatorname{Gal}\left(E^{\prime}\right)$ is an isomorphism by [FJ08, 16.10.8].

Definition 7.5. We say that $F$ is $\mathbf{P S C C}$ if it satisfies the following local-global principle for any smooth absolutely irreducible $F$-variety $V: V(F) \neq \emptyset$ iff $V\left(F^{\prime}\right) \neq \emptyset$ for all $F^{\prime} \in \mathrm{CC}_{\mathfrak{p}}(F), \mathfrak{p} \in \mathfrak{S}$.

Definition 7.6. A prime $\mathfrak{P}$ is quasi-local if it is an ordering or a $p$-valuation with value group a $\mathbb{Z}$-group, cf. [Feh13, Def. 3.5, Rem. 3.6], and $F$ is $\mathfrak{S}$-quasi-local if all $\mathfrak{P} \in \mathcal{S}_{\mathfrak{p}}(F)$, $\mathfrak{p} \in \mathfrak{S}$, are quasi-local. The field $F$ is $\mathfrak{S}$-SAP if it satisfies the strong approximation property of [Feh13, Def. 10.1]. (We will not make use of the precise definition.)

Proposition 7.7. If $F / K$ is algebraic, or $F$ is PSCC, then $F$ is $\mathfrak{S}$-quasi-local and S-SAP.

Proof. See [Feh13, Lemma 4.8, Prop. 4.9, Lemma 10.5, Prop. 10.7].

Definition 7.8. An extension $M / F$ is totally $\mathfrak{S}$-adic if the restriction map $\mathcal{S}_{\mathfrak{p}}(M) \rightarrow$ $\mathcal{S}_{\mathfrak{p}}(F),\left.\mathfrak{P} \mapsto \mathfrak{P}\right|_{F}$, is surjective for all $\mathfrak{p} \in \mathfrak{S}$, cf. [Feh13, Def. 11.1]. For $\mathfrak{p} \in \mathfrak{S}$ we let $R_{\mathfrak{p}}(F)=\bigcap_{\mathfrak{P} \in \mathcal{S}_{\mathfrak{p}}(F)} \mathcal{O}_{\mathfrak{P}}$, where $\mathcal{O}_{\mathfrak{P}}$ is the positive cone resp. the valuation ring of $\mathfrak{P}$, cf. [Feh13, Def. 4.2].

Proposition 7.9. If $F$ is $\mathfrak{S}-S A P$, then the following statements are equivalent for every extension $M / F$ :

(1) $M / F$ is totally $\mathfrak{S}$-adic.

(2) $R_{\mathfrak{p}}(M) \cap F=R_{\mathfrak{p}}(F)$ for every $\mathfrak{p} \in \mathfrak{S}$.

(3) $R_{\mathfrak{p}}(M) \cap F \subseteq R_{\mathfrak{p}}(F)$ for every $\mathfrak{p} \in \mathfrak{S}$.

Proof. See [Feh13, Lemma 11.4].

We now recall some results on the model theory of PSCC fields.

Definition 7.10. Let $\mathcal{L}_{\text {ring }}=\{+,-, \cdot, 0,1\}$ be the language of rings, $\mathcal{L}_{\text {ring, } \mathfrak{S}}=\mathcal{L}_{\text {ring }} \cup\left\{R_{\mathfrak{p}}\right.$ : $\mathfrak{p} \in \mathfrak{S}\}$, where each $R_{\mathfrak{p}}$ is a unary predicate symbol, and $\mathcal{L}_{\text {ring, } \mathfrak{p}}=\mathcal{L}_{\text {ring, }\{\mathfrak{p}\}}$. For a language $\mathcal{L}$ we denote by $\mathcal{L}(K)=\mathcal{L} \cup\left\{c_{a}: a \in K\right\}$ the augmentation by constants from $K$.

Proposition 7.11 . There is a recursive $\mathcal{L}_{\text {ring }}(K)$-theory $T_{\mathrm{PSCC}}$ such that $F$ satisfies $T_{\mathrm{PSCC}}$ if and only if $F$ is PSCC.

Proof. See [Feh13, Prop. 9.3].

Proposition 7.12. For each $\mathfrak{p} \in \mathfrak{S}$ there exists an $\mathcal{L}_{\text {ring }}(K)$-formula $\varphi_{R, \mathfrak{p}}$ that defines $R_{\mathfrak{p}}(F)$ in $F$ for each $\mathrm{PSCC}$ field $F$.

Proof. See [Feh13, Thm. 1.2]. 
Proposition 7.13. For every $\mathfrak{p} \in \mathfrak{S}$ there exists a recursive map $\varphi(\mathbf{x}) \mapsto \hat{\varphi}_{\mathfrak{p}, \exists}(\mathbf{x})$ from $\mathcal{L}_{\text {ring-formulas to }} \mathcal{L}_{\text {ring,p }}(K)$-formulas such that for $F \supseteq K$ which is $\mathfrak{S}$-quasi-local and $a_{1}, \ldots, a_{m} \in F$, one has $\left(F, R_{\mathfrak{p}}(F)\right) \models \hat{\varphi}_{\mathfrak{p}, \exists}(\mathbf{a})$ iff $F^{\prime} \models \varphi(\mathbf{a})$ for some $F^{\prime} \in \mathrm{CC}_{\mathfrak{p}}(F)$.

Proof. Apply [Feh13, Lemma 8.3] to $\neg \varphi(\mathbf{x})$.

Proposition 7.14. If $F$ is $\mathrm{PSCC}$ and $F \prec M$ is an elementary extension, then $M / F$ is regular and totally $\mathfrak{S}$-adic.

Proof. See [Feh13, Cor. 11.5].

The following embedding theorem will play a central role in Section 10 .

Proposition 7.15 (Pop). Let $L \supseteq K$ and let $E / L, F / L$ be regular extensions, where $E$ is countable and $F$ is $\aleph_{1}$-saturated and $\mathrm{PSCC}$. Then for every homomorphism $\gamma: \operatorname{Gal}(F) \rightarrow$ $\operatorname{Gal}(E)$ with $\operatorname{res}_{\tilde{E} / \tilde{L}} \circ \gamma=\left.\operatorname{res}_{\tilde{F} / \tilde{L}}\right|_{\operatorname{Gal}(F)}$, there exists an L-embedding $\tilde{E} \rightarrow \tilde{F}$ such that $\gamma(\tau)=\left.\tau\right|_{\tilde{E}}$ for all $\tau \in \operatorname{Gal}(F)$.

Proof. This follows from [Pop86, 6.1], as PSCC fields are pseudo classically closed, see also [Feh13, Prop. 4.6]. A proof in the present setting with all details can be found in Feh10, 2.11.5].

\section{S-Adic Absolute Galois Group Piles}

We now define the group pile $\operatorname{Gal}_{\mathfrak{S}}(F)$ and prove some of its basic properties.

Definition 8.1. The S-adic absolute Galois group pile of $F$ is the group pile

$$
\operatorname{Gal}_{\mathfrak{S}}(F)=\left(\operatorname{Gal}(F), \mathcal{G}_{\mathfrak{p}}\right)_{\mathfrak{p} \in \mathfrak{S}},
$$

where $\mathcal{G}_{\mathfrak{p}}=\left\{\operatorname{Gal}\left(F^{\prime}\right): F^{\prime} \in \mathrm{CC}_{\mathfrak{p}}(F)\right\}$. For a Galois extension $E / F$, let $\operatorname{Gal}_{\mathfrak{S}}(E / F)=$ $\operatorname{Gal}_{\mathfrak{S}}(F) / \mathrm{Gal}(E)$ be the $\mathfrak{S}$-adic Galois group pile of $E / F$.

In order to prove that $\operatorname{Gal}_{\mathfrak{S}}(F)$ is a indeed group pile, we will make use of the following group theoretical lemma:

Lemma 8.2. Let $G$ be a profinite group and $\Gamma$ a finitely generated profinite group. Then $\mathcal{G}=\{H \leq G: H$ is a quotient of $\Gamma\} \subseteq \operatorname{Subgr}(G)$ is closed.

Proof. We prove that $\operatorname{Subgr}(G) \backslash \mathcal{G}$ is open. Let $H \leq G$ such that $H$ is not a quotient of $\Gamma$. Since $\Gamma$ is finitely generated, by [FJ08, 16.10.7(a)] there exists an open normal subgroup $H_{0} \triangleleft H$ such that $H / H_{0}$ is not a quotient of $\Gamma$. Let $N \triangleleft G$ be an open normal subgroup with $N \cap H \leq H_{0}$. Since $H / H_{0}$ is not a quotient of $\Gamma$, also $H /(N \cap H)$ is not a quotient of $\Gamma$. If $H^{\prime} \leq G$ and $H^{\prime} N=H N$, then $H^{\prime} /\left(N \cap H^{\prime}\right) \cong H^{\prime} N / N \cong H /(N \cap H)$, hence $H^{\prime}$ is not a quotient of $\Gamma$. The set of such $H^{\prime}$ forms an open neighborhood of $H$.

The following statement is similar to [HJP09, Lemma 10.3(c)-(d)], which, however, is concerned with fields instead of group piles, and is restricted to certain subfields of $K^{\mathfrak{S}}$.

Proposition 8.3. The $\mathfrak{S}$-adic absolute Galois group pile $\mathbf{G a l}_{\mathfrak{S}}(F)$ is a separated reduced deficient group pile.

Proof. Let $\mathbf{G}=\left(G, \mathcal{G}_{\mathfrak{p}}\right)_{\mathfrak{p} \in \mathfrak{S}}=\operatorname{Gal}_{\mathfrak{S}}(F)$.

We first prove that $\mathbf{G}$ is a group pile. Let $\operatorname{Gal}_{\mathfrak{S}}(K)=\left(H, \mathcal{H}_{\mathfrak{p}}\right)_{\mathfrak{p} \in \mathfrak{S}}$ and fix $\mathfrak{p} \in \mathfrak{S}$. We have to show that $\mathcal{G}_{\mathfrak{p}}$ is closed in $\operatorname{Subgr}(G)$. Let $\Gamma=\operatorname{Gal}\left(K_{\mathfrak{p}}\right)$. Since $\mathfrak{p}$ is local, we have $\mathcal{H}_{\mathfrak{p}}=\Gamma^{H}$, cf. [Feh13, Rem. 3.6], hence $\mathcal{H}_{\mathfrak{p}}$ is closed in $\operatorname{Subgr}(H)$. By Proposition A.3 and Proposition B.5, $\Gamma$ is finitely generated. 
Let $G_{0} \leq G$. We claim that $G_{0} \in \mathcal{G}_{\mathfrak{p}}$ if and only if $G_{0}$ is a quotient of $\Gamma$ and $\operatorname{res}_{\tilde{F} / \tilde{K}}\left(G_{0}\right) \in$ $\mathcal{H}_{\mathfrak{p}}$. Indeed, if $G_{0} \in \mathcal{G}_{\mathfrak{p}}$, then $\operatorname{res}_{\tilde{F} / \tilde{K}}\left(G_{0}\right) \in \mathcal{H}_{\mathfrak{p}}$ and $G_{0} \cong \operatorname{res}_{\tilde{F} / \tilde{K}}\left(G_{0}\right) \cong \Gamma$ by Lemma 7.4 . Conversely, if $\operatorname{res}_{\tilde{F} / \tilde{K}}\left(G_{0}\right) \in \mathcal{H}_{\mathfrak{p}}=\Gamma^{H}$, then $\Gamma$ is quotient of $G_{0}$. Hence, if also $G_{0}$ is a quotient of $\Gamma$, then $G_{0} \cong \Gamma$ by [FJ08, 16.10.7]. Therefore, by Proposition A.3 and Proposition B.6, the fixed field $F^{\prime}$ of $G_{0}$ is real closed resp. $p$-adically closed of the same type as $\mathfrak{p}$. In addition, $\operatorname{res}_{\tilde{F} / \tilde{K}}\left(G_{0}\right) \in \mathcal{H}_{\mathfrak{p}}$ implies that $F^{\prime} \in \mathrm{CC}_{\mathfrak{p}}(F)$, i.e. $G_{0} \in \mathcal{G}_{\mathfrak{p}}$.

By Lemma 8.2, the set of $G_{0} \leq G$ such that $G_{0}$ is a quotient of $\Gamma$ is closed. Since $\mathcal{H}_{\mathfrak{p}}=\Gamma^{H}$ is closed, and $\operatorname{res}_{\tilde{F} / \tilde{K}}: \operatorname{Subgr}(G) \rightarrow \operatorname{Subgr}(H)$ is continuous by Lemma 3.2, the set of $G_{0} \leq G$ with $\operatorname{res}_{\tilde{F} / \tilde{K}}\left(G_{0}\right) \in \mathcal{H}_{\mathfrak{p}}$ is closed. Therefore, $\mathcal{G}_{\mathfrak{p}}$ is closed.

We now prove that $\mathbf{G}$ is separated and reduced. Let $\mathfrak{p}, \mathfrak{q} \in \mathfrak{S}, \Gamma \in \mathcal{G}_{\mathfrak{p}}, \Gamma_{1} \in \mathcal{G}_{\mathfrak{q}}$, and assume that $\Gamma \subseteq \Gamma_{1}$.

If $\mathfrak{p}$ or $\mathfrak{q}$ is an ordering, then both are orderings and $\Gamma=\Gamma_{1}$, since the absolute Galois group of a real closed field is finite (Proposition A.3), and the absolute Galois group of a $p$-adically closed field is non-trivial and torsion-free (Proposition B.5). So since the ordering of a real closed field is unique, $\mathfrak{p}=\mathfrak{q}$.

If $\mathfrak{p}$ is a $p$-valuation and $\mathfrak{q}$ is a $q$-valuation, let $F^{\prime}$ and $F_{1}^{\prime}$ be the fixed fields of $\Gamma$ resp. $\Gamma_{1}$, and let $K^{\prime}=\tilde{K} \cap F^{\prime}$ and $K_{1}^{\prime}=\tilde{K} \cap F_{1}^{\prime}$. Then $K_{1}^{\prime} \subseteq K^{\prime}$, and $K^{\prime} \in \mathrm{CC}_{\mathfrak{p}}(K)$ and $K_{1}^{\prime} \in \mathrm{CC}_{\mathfrak{q}}(K)$ by Lemma 7.4. Thus, since $\mathfrak{p}$ and $\mathfrak{q}$ are local, $K^{\prime}$ is Henselian with respect to two rank one valuations, which must be equivalent by F. K. Schmidt's theorem, cf. [EP05, 4.4.1]. In particular, $p=q$. Thus the restriction of the unique $p$-valuation of $F^{\prime}$ to $F_{1}^{\prime}$ is the unique $p$-valuation of $F_{1}^{\prime}$, so $\mathfrak{p}=\mathfrak{q}$. Therefore, by the maximality of $p$-adically closed fields (of the same type), $F^{\prime}=F_{1}^{\prime}$, hence $\Gamma=\Gamma_{1}$.

Remark 8.4. If $\left(N_{i}\right)_{i \in I}$ is a directed family of closed normal subgroups of a group pile $\mathbf{G}$ with $\bigcap_{i \in I} N_{i}=1$, then $\mathbf{G} \cong \lim _{i \in I} \mathbf{G} / N_{i}$. In particular, $\operatorname{Gal}_{\mathfrak{S}}(F)=\lim _{E} \operatorname{Gal}_{\mathfrak{S}}(E / F)$, where $E$ ranges over all finite Galois extensions of $F$.

Lemma 8.5. Let $F \supseteq K$ be $\mathfrak{S}$-quasi-local, let $M / F$ be an extension, and let

$$
\operatorname{res}_{\tilde{M} / \tilde{F}}: \mathbf{G a l}_{\mathfrak{S}}(M) \rightarrow \mathbf{G a l}_{\mathfrak{S}}(F)
$$

be the restriction map. Then $\operatorname{res}_{\tilde{M} / \tilde{F}}$ is a homomorphism of group piles, and the following are equivalent:

(1) $\operatorname{res}_{\tilde{M} / \tilde{F}}$ is an epimorphism.

(2) $\operatorname{res}_{\tilde{M} / \tilde{F}}$ is a rigid epimorphism.

(3) $M / F$ is regular and totally $\mathfrak{S}$-adic.

Proof. Let $\mathfrak{p} \in \mathfrak{S}$. If $M^{\prime} \in \mathrm{CC}_{\mathfrak{p}}(M)$, then $F^{\prime}=M^{\prime} \cap \tilde{F} \in \mathrm{CC}_{\mathfrak{p}}(F)$ by Lemma 7.4. Thus, $\operatorname{res}_{\tilde{M} / \tilde{F}}: \operatorname{Gal}(M) \rightarrow \operatorname{Gal}(F)$ indeed induces a homomorphism of group piles $\operatorname{res}_{\tilde{M} / \tilde{F}}$ : $\operatorname{Gal}_{\mathfrak{S}}(M) \rightarrow \operatorname{Gal}_{\mathfrak{S}}(F)$.

Proof of $(1) \Rightarrow(3)$ : Suppose that $\operatorname{res}_{\tilde{M} / \tilde{F}}: \operatorname{Gal}_{\mathfrak{S}}(M) \rightarrow \operatorname{Gal}_{\mathfrak{S}}(F)$ is an epimorphism of group piles. Then $\operatorname{res}_{\tilde{M} / \tilde{F}}: \operatorname{Gal}(M) \rightarrow \operatorname{Gal}(F)$ is surjective, so $M / F$ is regular. Let $\mathfrak{p} \in \mathfrak{S}$, $\mathfrak{P} \in \mathcal{S}_{\mathfrak{p}}(F)$, and $F^{\prime} \in \mathrm{CC}(F, \mathfrak{P})$. Then there exists $M^{\prime} \in \mathrm{CC}_{\mathfrak{p}}(M)$ with $M^{\prime} \cap \tilde{F}=F^{\prime}$. Let $\mathfrak{Q}^{\prime}$ be the unique prime of $M^{\prime}$ lying over $\mathfrak{p}$ and let $\mathfrak{Q}=\left.\mathfrak{Q}^{\prime}\right|_{M}$. Then $\mathfrak{Q} \in \mathcal{S}_{\mathfrak{p}}(M)$ and $\left.\mathfrak{Q}\right|_{F}=\mathfrak{P}$. Therefore, $M / F$ is totally $\mathfrak{S}$-adic.

Proof of $(3) \Rightarrow(2)$ : Since $M / F$ is regular, $\operatorname{res}_{\tilde{M} / \tilde{F}}: \operatorname{Gal}(M) \rightarrow \operatorname{Gal}(F)$ is surjective. Consider $\mathfrak{p} \in \mathfrak{S}, \mathfrak{P} \in \mathcal{S}_{\mathfrak{p}}(F)$, and $F^{\prime} \in \mathrm{CC}(F, \mathfrak{P})$. Since $M / F$ is totally $\mathfrak{S}$-adic, there exists $\mathfrak{Q} \in \mathcal{S}_{\mathfrak{p}}(M)$ lying over $\mathfrak{P}$. If $M^{\prime \prime} \in \mathrm{CC}(M, \mathfrak{Q})$, then $F^{\prime \prime}=M^{\prime \prime} \cap \tilde{F} \in \mathrm{CC}(F, \mathfrak{P})$ by Lemma 7.4. Since $\mathfrak{P}$ is quasi-local, $F^{\prime}$ and $F^{\prime \prime}$ are conjugate over $F$, cf. [Feh13, Rem 3.6]. Since $\operatorname{res}_{\tilde{M} / \tilde{F}}$ is surjective, there exists a conjugate $M^{\prime} \in \operatorname{CC}(M, \mathfrak{Q})$ of $M^{\prime \prime}$ 
with $M^{\prime} \cap \tilde{F}=F^{\prime}$. Therefore, $\operatorname{res}_{\tilde{M} / \tilde{F}}: \operatorname{Gal}_{\mathfrak{S}}(M) \rightarrow \mathbf{G a l}_{\mathfrak{S}}(F)$ is an epimorphism of group piles. By Lemma 7.4, res: $\operatorname{Gal}\left(M^{\prime}\right) \rightarrow \operatorname{Gal}\left(F^{\prime}\right)$ is an isomorphism, so $\operatorname{res}_{\tilde{M} / \tilde{F}}$ is rigid.

Proof of $(2) \Rightarrow(1)$ : This is trivial.

We now explain how to interpret statements about $\operatorname{Gal}_{\mathfrak{S}}(F)$ in $F$. Due to lack of a suitable reference we present the classical case $\mathfrak{S}=\emptyset$ in Appendix $\mathbb{C}$. Here we only explain how to extend this to general $\mathfrak{S}$. The following proposition generalizes Proposition C.9.

Proposition 8.6. To every ranked set of coformulas $\Sigma$ in the variables $v_{1}, v_{2}, \ldots$ we can assign recursively a set $\Sigma_{\text {ring }}$ of $\mathcal{L}_{\text {ring, }}$-formulas such that for every $F \supseteq K$ which is $\mathfrak{S}$-quasi-local, $\Sigma$ is cosatisfied in $\mathbf{G a l}_{\mathfrak{S}}(F)$ if and only if $\Sigma_{\text {ring }}$ is satisfied in $\left(F, R_{\mathfrak{p}}(F)\right)_{\mathfrak{p} \in \mathfrak{S}}$.

Proof. Building on the case $\mathfrak{S}=\emptyset$, we only have to explain how to translate statements of the form $\left(v_{1}, \ldots, v_{n}\right) \in \mathcal{G}_{\mathfrak{p}, n}$. Let $\operatorname{Gal}_{\mathfrak{S}}(F)=\left(G, \mathcal{G}_{\mathfrak{p}}\right)_{\mathfrak{p} \in \mathfrak{S}}, N=\operatorname{Gal}(E) \triangleleft G$ an open subgroup, and $\Delta N / N \in \operatorname{Subgr}(G / N)$. By definition, $\Delta N / N \in \mathcal{G}_{\mathfrak{p}, N}$ if and only if there is some $F^{\prime} \in \mathrm{CC}_{\mathfrak{p}}(F)$ such that, with $\Gamma=\operatorname{Gal}\left(F^{\prime}\right) \in \mathcal{G}_{\mathfrak{p}}, \Gamma N / N=\Delta N / N$. So $\left(x_{1} N_{1}, \ldots, x_{n} N_{n}\right) \in \mathcal{G}_{\mathfrak{p}, n}$ if and only if $N_{1}=\cdots=N_{n}$ and $H=\left\{x_{1} N_{1}, \ldots, x_{n} N_{n}\right\}$ is a subgroup of $G / N_{1}=\operatorname{Gal}(E / F)$ that corresponds to a field $F \subseteq E^{\prime} \subseteq E$ that is the intersection of some $F^{\prime} \in \mathrm{CC}_{\mathfrak{p}}(F)$ with $E$. This is equivalent to the fact that every polynomial $f \in F[X]$ of degree bounded by $[E: F]$ that has a root in $E$ has a root in $F^{\prime}$ if and only if it has a root in $E^{\prime}$. By Proposition 7.13 , the existence of such an $F^{\prime}$ can be expressed by an $\mathcal{L}_{\text {ring, }, \mathfrak{S}}$-formula in $\left(F, R_{\mathfrak{p}}(F)\right)_{\mathfrak{p} \in \mathfrak{S}}$.

Corollary 8.7. There is a recursive map $\varphi \mapsto \varphi_{\text {ring }}$ from cosentences to $\mathcal{L}_{\text {ring }}(K)$ sentences such that for every $\mathrm{PSCC}$ field $F$ and every cosentence $\varphi$, we have that $F \models \varphi_{\text {ring }}$ iff $S\left(\mathbf{G a l}_{\mathfrak{S}}(F)\right) \models \varphi$.

Proof. A PSCC field $F$ is $\mathfrak{S}$-quasi-local (Proposition 7.7 ) and the $R_{\mathfrak{p}}(F)$ are $K$-definable (Proposition 7.12) by some formula $\varphi_{R, \mathfrak{p}}$, so the claim follows from the special case $\Sigma=$ $\{\varphi\}$ of Proposition 8.6 by replacing all occurrences of the predicates $R_{\mathfrak{p}}$ by $\varphi_{R, \mathfrak{p}}$.

Corollary 8.8. If $F$ is $\mathrm{PSCC}$ and $\aleph_{1}$-saturated, then $\mathbf{G a l}_{\mathfrak{S}}(F)$ is $\aleph_{1}$-cosaturated.

Proof. This follows from Proposition 8.6 just like Corollary C.11 follows from Proposition C.9. using, like in the proof of the previous corollary, Proposition 7.7 and Proposition 7.12 .

Definition 8.9. If we apply Corollary 8.7 to the sentences of the cotheory $T_{\mathrm{C}, \mathfrak{S}, e}^{\mathrm{co}}$ (Definition 6.8), we get an $\mathcal{L}_{\text {ring }}(K)$-theory, which we denote by $T_{\mathrm{C}, \mathfrak{S}, e}^{\text {ring }}$.

\section{SUBFIELDS OF $K^{\mathfrak{S}}$}

We now turn to the fields $K^{\mathfrak{S}}(\boldsymbol{\sigma})$ mentioned in the introduction. We can define $K^{\mathfrak{S}}=$ $\bigcap_{\mathfrak{p} \in \mathfrak{S}} \cap \mathrm{CC}_{\mathfrak{p}}(K)$, cf. [GJ02, 0.1], [HJP09].

Lemma 9.1. Let $K \subseteq L \subseteq K^{\mathfrak{S}}$ be a field and let $\mathfrak{p} \in \mathfrak{S}$. Then the following holds:

(1) $L / K$ is totally $\mathfrak{S}$-adic.

(2) $\mathrm{CC}_{\mathfrak{p}}(L)=\mathrm{CC}_{\mathfrak{p}}(K)$

(3) $R_{\mathfrak{p}}(L)=L \cap R_{\mathfrak{p}}\left(K^{\mathfrak{S}}\right)=L \cap \bigcap_{K^{\prime} \in \mathrm{CC}_{\mathfrak{p}}(K)} R_{\mathfrak{p}}\left(K^{\prime}\right)$

Proof. (1): For $\mathfrak{p} \in \mathfrak{S}$, take $K^{\prime} \in \mathrm{CC}_{\mathfrak{p}}(K)$ and $\mathfrak{P} \in \mathcal{S}_{\mathfrak{p}}\left(K^{\prime}\right)$. Since $L \subseteq K^{\mathfrak{S}} \subseteq K^{\prime}$ we can restrict $\mathfrak{P}$ to $\left.\mathfrak{P}\right|_{L} \in \mathcal{S}_{\mathfrak{p}}(L)$, which lies over $\mathfrak{p}$.

(2): Since $L / K$ is algebraic, it is clear that $\mathrm{CC}_{\mathfrak{p}}(L) \subseteq \mathrm{CC}_{\mathfrak{p}}(K)$. Conversely, if $K^{\prime} \in$ $\mathrm{CC}_{\mathfrak{p}}(K)$, then $L \subseteq K^{\mathfrak{S}} \subseteq K^{\prime}$ and therefore $K^{\prime} \in \mathrm{CC}_{\mathfrak{p}}(L)$. 
(3): By $(2), \mathrm{CC}_{\mathfrak{p}}(L)=\mathrm{CC}_{\mathfrak{p}}(K)=\mathrm{CC}_{\mathfrak{p}}\left(K^{\mathfrak{S}}\right)$. Thus,

$$
\begin{aligned}
R_{\mathfrak{p}}(L) & =\bigcap_{\mathfrak{P} \in \mathcal{S}_{\mathfrak{p}}(L)} \mathcal{O}_{\mathfrak{P}}=\bigcap_{L^{\prime} \in \mathrm{CC}_{\mathfrak{p}}(L)} R_{\mathfrak{p}}\left(L^{\prime}\right) \cap L=L \cap \bigcap_{K^{\prime} \in \mathrm{CC}_{\mathfrak{p}}(K)} R_{\mathfrak{p}}\left(K^{\prime}\right) \\
& =L \cap \bigcap_{K^{\prime} \in \mathrm{CC}_{\mathfrak{p}}\left(K^{\mathfrak{S}}\right)} R_{\mathfrak{p}}\left(K^{\prime}\right) \cap K^{\mathfrak{S}}=L \cap \bigcap_{\mathfrak{P} \in \mathcal{S}_{\mathfrak{p}}\left(K^{\mathfrak{S}}\right)} \mathcal{O}_{\mathfrak{P}}=L \cap R_{\mathfrak{p}}\left(K^{\mathfrak{S}}\right) .
\end{aligned}
$$

Lemma 9.2. Let $K \subseteq E \subseteq F \subseteq K^{\mathfrak{S}}$. If $E$ is $\mathrm{PSCC}$, then $F$ is $\mathrm{PSCC}$.

Proof. 3 Let $V$ be a smooth absolutely irreducible variety defined over $F$ with $V\left(F^{\prime}\right) \neq \emptyset$ for all $F^{\prime} \in \mathrm{CC}_{\mathfrak{p}}(F), \mathfrak{p} \in \mathfrak{S}$. Since by Proposition A.4 and Proposition B.2, $V\left(F^{\prime}\right)$ is in fact Zariski-dense in $V$ for all $F^{\prime} \in \mathrm{CC}_{\mathfrak{p}}(F), \mathfrak{p} \in \mathfrak{S}$, we can assume without loss of generality that $V$ is affine. Since $F / E$ is algebraic, $V$ is defined over a finite subextension $F_{0}$ of $F / E$. Let $W=\operatorname{res}_{F_{0} / E}(V)$ be the Weil restriction of $V$ and let $F_{1}$ be the Galois closure of $F_{0} / E$. Then $W$ is a variety defined over $E$ and there are $\sigma_{1}, \ldots, \sigma_{n} \in \operatorname{Gal}(E)$ with $\sigma_{1}=\operatorname{id}_{\tilde{E}}$ such that $W$ is isomorphic over $F_{1}$ to $\prod_{i=1}^{n} V^{\sigma_{i}}$, and the projection onto the first factor $W \rightarrow V^{\sigma_{1}}=V$ is defined over $F_{0}$, cf. [FJ08, 10.6.2]. Since $V$ is smooth, also $W$ is smooth.

Since $E \subseteq F \subseteq K^{\mathfrak{S}}$ and $E \subseteq F_{1} \subseteq K^{\mathfrak{S}}$, Lemma 9.1(2) implies that $\mathrm{CC}_{\mathfrak{p}}(E)=$ $\mathrm{CC}_{\mathfrak{p}}(F)=\mathrm{CC}_{\mathfrak{p}}\left(F_{1}\right)$ for all $\mathfrak{p} \in \mathfrak{S}$. In particular, if $E^{\prime} \in \mathrm{CC}_{\mathfrak{p}}(E)$, then $F_{1} \subseteq E^{\prime}$. Let $E^{\prime} \in \mathrm{CC}_{\mathfrak{p}}(E)$. Then $\sigma_{i}^{-1}\left(E^{\prime}\right) \in \mathrm{CC}_{\mathfrak{p}}(E)=\mathrm{CC}_{\mathfrak{p}}(F)$, so $V\left(\sigma_{i}^{-1}\left(E^{\prime}\right)\right) \neq \emptyset$ by assumption. Thus $V^{\sigma_{i}}\left(E^{\prime}\right) \neq \emptyset$ for all $i$, and therefore $W\left(E^{\prime}\right) \neq \emptyset$, as $F_{1} \subseteq E^{\prime}$. Since $E$ is PSCC, $W(E) \neq \emptyset$, so in particular $W(F) \neq \emptyset$. Hence, since $F_{0} \subseteq F$, it follows that $V(F) \neq \emptyset$, as claimed.

Definition 9.3. If $\boldsymbol{\sigma}=\left(\sigma_{1}, \ldots, \sigma_{e}\right) \in \operatorname{Gal}(K)^{e}$, we denote by $\tilde{K}(\boldsymbol{\sigma})$ the fixed field of the group $\left\langle\sigma_{1}, \ldots, \sigma_{e}\right\rangle \leq \operatorname{Gal}(K)$ in $\tilde{K}$, and let $K^{\mathfrak{S}}(\boldsymbol{\sigma})=K^{\mathfrak{S}} \cap \tilde{K}(\boldsymbol{\sigma})$. We say that a statement holds for almost all $\boldsymbol{\sigma} \in \operatorname{Gal}(K)^{e}$ if the set of those $\boldsymbol{\sigma} \in \operatorname{Gal}(K)^{e}$ for which it holds has measure 1 with respect to the unique Haar probability measure on $\operatorname{Gal}(K)^{e}$.

Proposition 9.4 (Geyer-Jarden). Let $\mathfrak{S}$ be a finite set of local primes of a countable Hilbertian field $K$ of characteristic zero, and let $e \geq 0$. Then for almost all $\boldsymbol{\sigma} \in \operatorname{Gal}(K)^{e}$, the field $K^{\mathfrak{S}}(\boldsymbol{\sigma})$ is PSCC.

Proof. By [GJ02, Theorem A], for almost all $\boldsymbol{\sigma} \in \mathrm{Gal}(K)^{e}$, the maximal Galois extension $M$ of $K$ inside $K^{\mathfrak{S}}(\boldsymbol{\sigma})$ satisfies a local-global principle with respect to the sets $\mathcal{S}_{\mathfrak{p}}^{\prime}(M)$ of orderings and arbitrary valuations on $M$ lying over $\mathfrak{p} \in \mathfrak{S}$. However, since $M / K$ is totally $\mathfrak{S}$-adic by Lemma 9.1(1), and all extensions of $\mathfrak{p}$ to $M$ are conjugate since $M / K$ is Galois, in fact $\mathcal{S}_{\mathfrak{p}}^{\prime}(M)=\mathcal{S}_{\mathfrak{p}}(M)$. In other words, $M$ is PSCC. Since $K \subseteq M \subseteq K^{\mathfrak{S}}(\boldsymbol{\sigma}) \subseteq K^{\mathfrak{S}}$, the claim follows from Lemma 9.2 .

Proposition 9.5 (Haran-Jarden-Pop). Let $\mathfrak{S}$ be a finite set of local primes of a countable Hilbertian field $K$ of characteristic zero, and let $e \geq 0$. Then for almost all $\boldsymbol{\sigma} \in \operatorname{Gal}(K)^{e}$, $\operatorname{Gal}_{\mathfrak{S}}\left(K^{\mathfrak{S}}(\boldsymbol{\sigma})\right)$ is isomorphic to the deficient reduct of the e-free semi-constant group pile of $\left(\operatorname{Gal}\left(K_{\mathfrak{p}}\right)\right)_{\mathfrak{p} \in \mathfrak{S}}$ over perfect profinite spaces $\left(T_{\mathfrak{p}}\right)_{\mathfrak{p} \in \mathfrak{S}}$.

Proof. This is proven in [HJP09]. Indeed, by [HJP09, Prop. 12.3], for almost all $\boldsymbol{\sigma} \in$ $\operatorname{Gal}(K)^{e}$, the field $M=K^{\mathfrak{S}}(\boldsymbol{\sigma})$ satisfies condition (1) of $\S 10$ of that work. In the proof of [HJP09, Prop. 11.2] it is proven that in this case $\operatorname{Gal}(M, \mathfrak{S}):=\left(G, \operatorname{Gal}(\tilde{K}(\boldsymbol{\sigma}))^{G}, \mathcal{G}_{\mathfrak{p}}\right)_{\mathfrak{p} \in \mathfrak{S}}$, where $\operatorname{Gal}_{\mathfrak{S}}(M)=\left(G, \mathcal{G}_{\mathfrak{p}}\right)_{\mathfrak{p} \in \mathfrak{S}}$, is a so called 'Cantor group pile over $\left(\operatorname{Gal}\left(K_{\mathfrak{p}}\right)\right)_{\mathfrak{p} \in \mathfrak{S}}$ '. By

\footnotetext{
${ }^{3}$ This proof corrects an inaccuracy in GJ02, Proof of Lemma 1.6 Part B].
} 
[HJP09, Cor. 6.2] and [HJP09, Prop. 6.3], every Cantor group pile over $\left(\operatorname{Gal}\left(K_{\mathfrak{p}}\right)\right)_{\mathfrak{p} \in \mathfrak{S}}$ is isomorphic to the group pile $\mathbf{G}_{T}$ of [HJP09, Prop. 5.3], which is exactly the $e$-free semi-

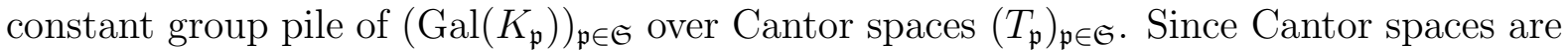
perfect and the deficient reduct of $\operatorname{Gal}(M, \mathfrak{S})$ is $\operatorname{Gal}_{\mathfrak{S}}(M)$, the claim follows.

\section{Axiomatization}

We now present the axiomatization of the theory of almost all $K^{\mathfrak{S}}(\boldsymbol{\sigma})$.

Setting 10.1. Let $\mathfrak{S}$ be a finite set of local primes of a countable Hilbertian field $K$ of characteristic zero.

Definition 10.2. For a set $S \subseteq \tilde{K}$ let $N_{K}(S)=\{f \in K[X]: f$ has no root in $S\}$.

Definition 10.3. Let the $\mathcal{L}_{\text {ring }}(K)$-theory $T_{\text {alg, } \mathfrak{S}}$ consist of the following sentences:

(1) For each $f \in N_{K}\left(K^{\mathfrak{S}}\right)$ the sentence

$$
\neg(\exists x)(f(x)=0) .
$$

(2) For each $\mathfrak{p} \in \mathfrak{S}$ and each $f \in N_{K}\left(R_{\mathfrak{p}}\left(K^{\mathfrak{S}}\right)\right)$ the sentence

$$
\neg(\exists x)\left(\varphi_{\mathrm{R}, \mathfrak{p}}(x) \wedge f(x)=0\right),
$$

where $\varphi_{\mathrm{R}, \mathfrak{p}}$ is the formula of Proposition 7.12 .

Lemma 10.4. A PSCC field $F \supseteq K$ is a model of $T_{\text {alg, } \mathfrak{S}}$ if and only if $F \cap \tilde{K} \subseteq K^{\mathfrak{S}}$ and $F / F \cap \tilde{K}$ is totally $\mathfrak{S}$-adic.

Proof. Since $F$ is PSCC, $\varphi_{\mathrm{R}, \mathfrak{p}}(F)=R_{\mathfrak{p}}(F)$ for each $\mathfrak{p} \in \mathfrak{S}$ by Proposition 7.12 . Let $L=F \cap \tilde{K}$. Since $L / K$ is algebraic, $L$ is $\mathfrak{S}$-SAP by Proposition 7.7 .

Suppose that $F$ satisfies $T_{\text {alg, } \mathfrak{S}}$. By (1), $L \subseteq K^{\mathfrak{S}}$. If $\mathfrak{p} \in \mathfrak{S}$, then $R_{\mathfrak{p}}(L)=R_{\mathfrak{p}}\left(K^{\mathfrak{S}}\right) \cap L$ by Lemma 9.1. Since $R_{\mathfrak{p}}\left(K^{\mathfrak{S}}\right)$ is $\operatorname{Gal}(K)$-invariant, $(2)$ implies that $R_{\mathfrak{p}}(F) \cap \tilde{K} \subseteq R_{\mathfrak{p}}\left(K^{\mathfrak{S}}\right)$. Therefore, $R_{\mathfrak{p}}(F) \cap L \subseteq R_{\mathfrak{p}}\left(K^{\mathfrak{S}}\right) \cap L=R_{\mathfrak{p}}(L)$, so $F / L$ is totally $\mathfrak{S}$-adic by Proposition 7.9,

Conversely, suppose that $L \subseteq K^{\mathfrak{S}}$ and $F / L$ is totally $\mathfrak{S}$-adic. Since $L \subseteq K^{\mathfrak{S}}, F$ satisfies (1). By Proposition 7.9, $R_{\mathfrak{p}}(F) \cap L=R_{\mathfrak{p}}(L)$. So since $R_{\mathfrak{p}}(F) \cap L=R_{\mathfrak{p}}(F) \cap \tilde{K}$ and $R_{\mathfrak{p}}(L)=R_{\mathfrak{p}}\left(K^{\mathfrak{S}}\right) \cap L$ by Lemma 9.1, $F$ satisfies $(2)$.

Definition 10.5. Let the $\mathcal{L}_{\text {ring }}(K)$-theory $T_{\text {tot, }, e}$ consist of the following axioms:

(0) The axioms for fields and the positive diagram of $K$, cf. [FJ08, 7.3.1].

(1) The theory $T_{\mathrm{PSCC}}$ (Proposition 7.11).

(2) The theory $T_{\mathrm{C}, \mathfrak{S}, e}^{\text {ring }}$ (Definition 8.9).

(3) The theory $T_{\text {alg, } \mathfrak{S}}$ (Definition 10.3).

Lemma 10.6. A field $F \supseteq K$ is a model of $T_{\mathrm{tot}, \mathfrak{S}, e}$ if and only if it satisfies the following conditions:

(1) $F$ is PSCC.

(2) $\operatorname{Gal}_{\mathfrak{S}}(F)$ is an e-free $C$-pile.

(3) $F \cap \tilde{K} \subseteq K^{\mathfrak{S}}$ and $F / F \cap \tilde{K}$ is totally $\mathfrak{S}$-adic.

Proof. See Proposition 7.11 for (1), Proposition 6.9 and Corollary 8.7 for (2), and Lemma 10.4 for $(3)$.

Lemma 10.7. Let $K \subseteq L \subseteq E, F$ be fields such that the following conditions are satisfied.

(1) $E$ and $F$ are models of $T_{\text {tot, }, e}$.

(2) $E / L$ and $F / L$ are regular and totally $\mathfrak{S}$-adic.

(3) $E$ is countable and $F$ is $\aleph_{1}$-saturated. 
(4) $L$ is $\mathfrak{S}$-quasi-local.

Then there exists an L-embedding $i: E \rightarrow F$ with $F / i(E)$ regular and totally $\mathfrak{S}$-adic.

Proof. By (1), $E$ and $F$ are PSCC (Lemma 10.6(1)), so in particular S-quasi-local (Proposition [7.7). Let $\mathbf{G}=\mathbf{G a l}_{\mathfrak{S}}(F), \mathbf{B}=\mathbf{G a l}_{\mathfrak{S}}(E)$, and $\mathbf{A}=\mathbf{G a l}_{\mathfrak{S}}(L)$. Also by (1), $\mathbf{G}$ and $\mathbf{B}$ are $e$-free C-piles (Lemma 10.6(2)). By (2) and (4), the restriction maps $\operatorname{res}_{\tilde{F} / \tilde{L}}: \mathbf{G} \rightarrow \mathbf{A}$ and $\operatorname{res}_{\tilde{E} / \tilde{L}}: \mathbf{B} \rightarrow \mathbf{A}$ are rigid epimorphisms of group piles (Lemma 8.5). So $\left(\operatorname{res}_{\tilde{F} / \tilde{L}}, \operatorname{res}_{\tilde{E} / \tilde{L}}\right)$ is a rigid $e$-generated deficient embedding problem for $\mathbf{G}$.

By (3), $E$ is countable, so $\mathbf{B}$ has countable rank, and $F$ is $\aleph_{1}$-saturated, so $\operatorname{Gal}_{\mathfrak{S}}(F)$ is $\aleph_{1}$-cosaturated by Corollary 8.8. Hence, by Proposition 6.10 there exists an epimorphism $\gamma: \mathbf{G} \rightarrow \mathbf{B}$ such that $\operatorname{res}_{\tilde{E} / \tilde{L}}^{\circ} \gamma=\operatorname{res}_{\tilde{F} / \tilde{L}}$. By Proposition 7.15, this gives an $L$-embedding $i: E \rightarrow F$ such that $\gamma=\operatorname{res}_{\tilde{F} / \tilde{i(E)}}$. Hence, since $\gamma$ is an epimorphism of group piles, $F / i(E)$ is regular and totally $\mathfrak{S}$-adic by Lemma 8.5 .

The proof of the following proposition follows the proof of [FJ08, 20.3.3].

Proposition 10.8. Let $E, F \supseteq K$ be models of $T_{\text {tot, }, e, e}$ with $E \cap \tilde{K} \cong_{K} F \cap \tilde{K}$. Then $E \equiv_{K} F$, i.e. $E$ and $F$ are elementarily equivalent in $\mathcal{L}_{\text {ring }}(K)$.

Proof. Assume without loss of generality that $L:=E \cap \tilde{K}=F \cap \tilde{K}$. Let $E^{*}, F^{*}$ be $\aleph_{1}$-saturated elementary extensions of $E$ resp. $F$. By Lemma 10.6, the fields $E, F, E^{*}, F^{*}$ are PSCC, in particular also S-SAP (Proposition 7.7), and the extensions $E / L, F / L$, $E^{*} / L, F^{*} / L$ are regular and totally $\mathfrak{S}$-adic.

By Löwenheim-Skolem, there exists a countable elementary subfield $E_{0}$ of $E^{*}$ that contains $L$. Then also $E_{0} / L$ is regular and totally $\mathfrak{S}$-adic, and $E_{0}$ is a model of $T_{\text {tot, }, e, e}$, in particular PSCC and $\mathfrak{S}$-quasi-local (Proposition 7.7). Since $L / K$ is algebraic, $L$ is S-quasi-local (Proposition 7.7). Therefore, by Lemma 10.7, there exists an $L$-embedding $\alpha_{0}: E_{0} \rightarrow F^{*}$ with $F^{*} / \alpha_{0}\left(E_{0}\right)$ regular and totally $\mathfrak{S}$-adic.

Identify $E_{0}$ with $\alpha_{0}\left(E_{0}\right)$. Let $F_{0}$ be a countable elementary subfield of $F^{*}$ that contains

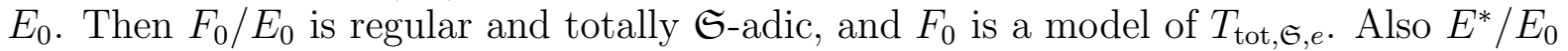
is regular and totally $\mathfrak{S}$-adic by Proposition 17.14. Hence, by Lemma 10.7, there is an $E_{0}$-embedding $\beta_{0}: F_{0} \rightarrow E^{*}$ with $E^{*} / \beta_{0}\left(F_{0}\right)$ regular and totally $\mathfrak{S}$-adic.

Now iterate this process to construct a tower of countable fields $E_{0} \subseteq F_{0} \subseteq E_{1} \subseteq F_{1} \subseteq$ ... such that each $E_{i}$ is an elementary subfield of $E^{*}$ and each $F_{i}$ is an elementary subfield of $F^{*}$. Then $M:=\bigcup_{i \in \mathbb{N}} E_{i}=\bigcup_{i \in \mathbb{N}} F_{i}$ is an elementary subfield of both $E^{*}$ and $F^{*}$, see for example [FJ08, 7.4.1(b)], so $E^{*} \equiv_{M} F^{*}$. In particular, $E^{*} \equiv_{K} F^{*}$, hence $E \equiv_{K} F$.

Lemma 10.9. If $F$ is a model of $T_{\mathrm{tot}, \mathfrak{S}, e}$, then $L:=F \cap \tilde{K} \subseteq K^{\mathfrak{S}}$ and $\operatorname{rk}\left(\operatorname{Gal}\left(K^{\mathfrak{S}} / L\right)\right) \leq e$. Proof. Let $\mathbf{G}=\mathbf{G a l}_{\mathfrak{S}}(F)$, and $\mathbf{A}=\mathbf{G a l}_{\mathfrak{S}}(L)$. By Lemma 10.6)(3), $L \subseteq K^{\mathfrak{S}}$ and $F / L$ is totally $\mathfrak{S}$-adic. Since $L / K$ is algebraic, $L$ is $\mathfrak{S}$-quasi-local (Proposition 7.7 ), so the restriction $\mathbf{G} \rightarrow \mathbf{A}$ is an epimorphism of group piles by Lemma 8.5. By Lemma 10.6(2), $\mathbf{G}$ is an $e$-free $\mathrm{C}$-pile. In particular, it is $e$-generated. Thus, by Lemma 3.15, also $\mathbf{A}$ is e-generated. By Lemma $9.1(2), \mathrm{CC}_{\mathfrak{p}}(L)=\mathrm{CC}_{\mathfrak{p}}\left(K^{\mathfrak{S}}\right)$, for all $\mathfrak{p} \in \mathfrak{S}$. Thus, $\mathbf{A}^{\prime}=$ $\operatorname{Gal}_{\mathfrak{S}}\left(K^{\mathfrak{S}}\right)^{\prime}$. But $\mathbf{G a l}_{\mathfrak{S}}\left(K^{\mathfrak{S}}\right)$ is self-generated by the definition of $K^{\mathfrak{S}}$, so $\mathbf{A}^{\prime}=\operatorname{Gal}_{\mathfrak{S}}\left(K^{\mathfrak{S}}\right)$. Therefore, $\operatorname{Gal}\left(K^{\mathfrak{S}} / L\right)=A / A^{\prime}=\bar{A}$ is generated by $e$ elements.

Definition 10.10. Let $T_{\text {almost, }, e}$ denote the set of all $\mathcal{L}_{\text {ring }}(K)$-sentences that are true in almost all fields $K^{\mathfrak{S}}(\boldsymbol{\sigma}), \boldsymbol{\sigma} \in \operatorname{Gal}(K)^{e}$.

The proof of the following result follows the proof of [FJ08, 20.5.4].

Theorem 10.11. The theory $T_{\mathrm{tot}, \mathfrak{S}, e}$ is an axiomatization of $T_{\text {almost, }, e}$, i.e. these two theories have the same models. 
Proof. First note that every model of $T_{\text {almost, } \mathfrak{S}_{e}}$ is a field containing $K$. By Definition 10.5(0), the same holds for every model of $T_{\text {tot, }, e, e}$. Next observe that almost all

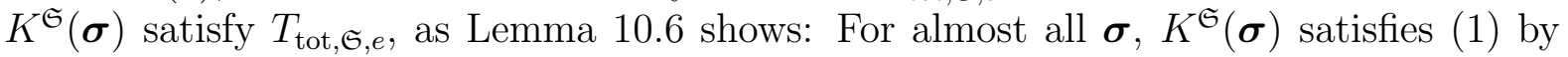
Proposition 9.4, (2) by Proposition 9.5 and Proposition 6.7, and (3) trivially. Thus, every model of $T_{\text {almost, }, e}$ is a model of $T_{\text {tot, }, \mathfrak{S}, e}$.

Conversely, let $E$ be a model of $T_{\text {tot, }, e, e}$ and let $L=E \cap \tilde{K}$. If we can construct a model $F$ of $T_{\text {almost, }, e, e}$ with $F \cap \tilde{K} \cong_{K} L$, then $E \equiv_{K} F$ by Proposition [10.8, so $E$ is a model of $T_{\text {almost, }, e}$ and we are done. Lemma 10.9 implies that $L \subseteq K^{\mathfrak{S}}$ and there exist $\tau_{1}, \ldots, \tau_{e} \in \operatorname{Gal}\left(K^{\mathfrak{S}} / K\right)$ that generate $\operatorname{Gal}\left(K^{\mathfrak{S}} / L\right)$. Let $\mathcal{N}$ be the set of finite Galois extensions of $K$ inside $K^{\mathfrak{S}}$. For each $N \in \mathcal{N}$, the set

$$
\begin{aligned}
\Sigma(N) & :=\left\{\boldsymbol{\sigma} \in \operatorname{Gal}(K)^{e}: \operatorname{res}_{\tilde{K} / N}\left(\sigma_{i}\right)=\operatorname{res}_{\tilde{K} / N}\left(\tau_{i}\right), i=1, \ldots, e\right\} \\
& \subseteq\left\{\boldsymbol{\sigma} \in \operatorname{Gal}(K)^{e}: K^{\mathfrak{S}}(\boldsymbol{\sigma}) \cap N \cong_{K} L \cap N\right\}
\end{aligned}
$$

has positive Haar measure. If $N_{1}, \ldots, N_{r} \in \mathcal{N}$, then $N_{1} \cdots N_{r} \in \mathcal{N}$ and $\Sigma\left(N_{1}\right) \cap \cdots \cap$ $\Sigma\left(N_{r}\right)=\Sigma\left(N_{1} \cdots N_{r}\right)$. Hence, by [FJ08, 7.6.1], there exists an ultrafilter $\mathcal{D}$ on $\operatorname{Gal}(K)^{e}$ which contains each of the sets $\Sigma(N), N \in \mathcal{N}$, and all sets of measure 1. Let

$$
F=\prod_{\boldsymbol{\sigma} \in \operatorname{Gal}(K)^{e}} K^{\mathfrak{S}}(\boldsymbol{\sigma}) / \mathcal{D}
$$

be the ultraproduct, and let $M=F \cap \tilde{K}$. Since $\mathcal{D}$ contains all sets of measure 1 , and almost all $K^{\mathfrak{S}}(\boldsymbol{\sigma})$ are models of $T_{\text {almost, }, e}, F$ is a model of $T_{\text {almost, }, e}$ by Łos' theorem. Furthermore, $M \subseteq K^{\mathfrak{S}}$, and $M \cap N \cong L \cap N$ for each $N \in \mathcal{N}$, since $\mathcal{D}$ contains $\Sigma(N)$. Therefore, $M \cong_{K} L$, see for example [FJ08, 20.6.3], as claimed.

\section{Decidability}

We now use the axiomatization of the previous section to prove the decidability of $T_{\text {almost, }, e}$. The method follows closely the proof of Jarden-Kiehne in FJ08, Chapter 20.6] for the special case $\mathfrak{S}=\emptyset$.

Definition 11.1. A set $X \subseteq \mathbb{N}^{n}$ is recursive if the characteristic function of $X$ is a recursive function, cf. [FJ08, Ch. 8.5]. If $\mathcal{L}$ is a countable language with a fixed embedding $\mathcal{L} \rightarrow \mathbb{N}$, then an $\mathcal{L}$-theory $T$ is decidable (or recursive) if the set $T$, identified with a subset of $\mathbb{N}$ via a Gödel numbering, is recursive, [FJ08, Ch. 8.6].

A presented field is a countable field $K$ together with an injection $\rho: K \rightarrow \mathbb{N}$ such that the images of the graphs of addition and multiplication are recursive. If $\mathcal{L}$ is a finite language containing $\mathcal{L}_{\text {ring }}$, then the injection $\rho: K \rightarrow \mathbb{N}$ induces an injection of the set of $\mathcal{L}(K)$-formulas into $\mathbb{N}$. We refer to this injection when we call an $\mathcal{L}(K)$-theory decidable.

If $K$ is a presented field, one can inject the ring of polynomials $K[X]$ into $\mathbb{N}$ via a recursive pairing function $\mathbb{N} \times \mathbb{N} \rightarrow \mathbb{N}$. We say that $K$ has a splitting algorithm if the set of irreducible polynomials in $K[X]$ is a recursive subset of $K[X]$. In that case, one can recursively factor elements of $K[X]$ into irreducible factors.

Definition 11.2. A prime $\mathfrak{p}$ of a presented field $\rho: K \rightarrow \mathbb{N}$ is recursive if the set $\rho\left(\mathcal{O}_{\mathfrak{p}}\right) \subseteq \mathbb{N}$ is recursive.

Setting 11.3. From now on, let $\mathfrak{S}$ be a finite set of recursive local primes of a presented countable Hilbertian field $K$ of characteristic zero that has a splitting algorithm.

Example 11.4. Every finite set of primes $\mathfrak{S}$ of a number field $K$ satisfies Setting 11.3: Choose any standard representation $K=\mathbb{Q}^{n} \hookrightarrow \mathbb{N}^{2 n} \hookrightarrow \mathbb{N}$ via recursive pairing functions. Then $K$ is countable, Hilbertian [FJ08, 13.4.2], and has a splitting algorithm [FJ08, 
19.1.3(b), 19.2.4], and every $\mathfrak{p} \in \mathfrak{S}$ is local and recursive. This last fact is well-known, but for lack of reference we sketch a proof: Since $\mathcal{O}_{\mathfrak{p}}$ is existentially definable in $K$ (see for example [Rum80, p. 212] for the archimedean and [Shl07, 4.2.4, 4.3.4] for the p-adic case), it is recursively enumerable. Since $K \backslash \mathcal{O}_{\mathfrak{p}}=-\mathcal{O}_{\mathfrak{p}} \backslash\{0\}$ in the archimedean and $K \backslash \mathcal{O}_{\mathfrak{p}}=\left(\pi \mathcal{O}_{\mathfrak{p}}\right)^{-1}$ in the $p$-adic case, where $\pi$ is a uniformizer at $\mathfrak{p}$, also $K \backslash \mathcal{O}_{\mathfrak{p}}$ is recursively enumerable, and so $\mathcal{O}_{\mathfrak{p}}$ is recursive.

Lemma 11.5. The sets $N_{K}\left(K^{\mathfrak{S}}\right)$ and $N_{K}\left(R_{\mathfrak{p}}\left(K^{\mathfrak{S}}\right)\right), \mathfrak{p} \in \mathfrak{S}$, are recursive.

Proof. Let $f=\sum_{i=0}^{n} a_{i} X^{i} \in K[X]$ be given. We have to decide whether $f$ has a root in $K^{\mathfrak{S}}$ resp. in $R_{\mathfrak{p}}\left(K^{\mathfrak{S}}\right)$. Using the splitting algorithm we can assume without loss of generality that $f$ is irreducible. Since $K^{\mathfrak{S}}=\bigcap_{\mathfrak{q} \in \mathfrak{S}} \bigcap \mathrm{CC}_{\mathfrak{q}}(K)$ and $R_{\mathfrak{p}}\left(K^{\mathfrak{S}}\right)=K^{\mathfrak{S}} \cap$ $\bigcap_{K^{\prime} \in \mathrm{CC}_{\mathfrak{p}}(K)} R_{\mathfrak{p}}\left(K^{\prime}\right)$ by Lemma $9.1(3)$, and all elements of $\mathrm{CC}_{\mathfrak{p}}(K)$ are $K$-conjugate, it suffices to decide whether $f$ has all roots in $K_{\mathfrak{q}}$ (resp. in $\left.R_{\mathfrak{q}}\left(K_{\mathfrak{q}}\right)\right)$ for all $\mathfrak{q} \in \mathfrak{S}$.

By [Feh13, Prop. 8.2] we can compute a universal $\mathcal{L}_{\text {ring, }}$-formula $\varphi_{1}$ such that $f$ has all roots in $K_{\mathfrak{q}}$ (resp. in $\left.R_{\mathfrak{q}}\left(K_{\mathfrak{q}}\right)\right)$ iff $\left(K, \mathcal{O}_{\mathfrak{q}}\right) \models \varphi_{1}(\mathbf{a})$. By applying the same result to the negation, we get a universal $\mathcal{L}_{\text {ring, } q}$-formula $\varphi_{2}$ such that $f$ has all roots in $K_{\mathfrak{q}}$ (resp. in $\left.R_{\mathfrak{q}}\left(K_{\mathfrak{q}}\right)\right)$ iff $\left(K, \mathcal{O}_{\mathfrak{q}}\right) \not \models \varphi_{2}(\mathbf{a})$. Therefore, since $\mathcal{O}_{\mathfrak{q}}$ is recursive, the set of such $f$ is both r.e. and co-r.e., hence recursive.

Lemma 11.6. The theory $T_{\text {tot, }, e}$ is recursive.

Proof. We check that the different sets of axioms in Definition 10.5 are recursive:

(0): Since $K$ is a presented field, the positive diagram of $K$ is recursive.

(1): In order to check that $T_{\mathrm{PSCC}}$ is recursive, we have to look into the definition [Feh13, Def. 9.1]. Part (1) of these axioms is recursive, because [Feh13, Def. 6.5] (1), $(3),(4),(5)$ consist of finitely many sentences, and (2) is recursive because $\mathcal{O}_{\mathfrak{p}}$ is. Part (2) of [Feh13, Def. 9.1] is recursive because the map $\eta_{n} \mapsto\left(\hat{\eta}_{n}\right)_{\mathfrak{p}, \forall}$ is recursive by [Feh13, Prop. 8.4].

(2): The theory $T_{\mathrm{C}, \mathfrak{S}, e}^{\mathrm{ring}}$ is recursive since $T_{\mathrm{C}, \mathfrak{S}, e}^{\mathrm{co}}$ is obviously recursive and the map $\varphi \mapsto \varphi_{\text {ring }}$ of Corollary 8.7 is recursive.

(3): Since $N_{K}\left(K^{\mathfrak{S}}\right)$ and $N_{K}\left(R_{\mathfrak{p}}\left(K^{\mathfrak{S}}\right)\right)$ are recursive by Lemma 11.5, the theory $T_{\text {alg, }}$ is recursive.

Definition 11.7. The set of test sentences is the smallest set of $\mathcal{L}_{\text {ring }}(K)$-sentences that contains all sentences of the form $(\exists x)(f(x)=0)$, where $f \in K[X]$ is a polynomial that completely decomposes over $K^{\mathfrak{S}}$, and is closed under negations, conjunctions, and disjunctions.

Lemma 11.8. Let $E, F \supseteq K$ be models of $T_{\mathrm{tot}, \mathfrak{S}, e}$. Then $E \equiv_{K} F$ if and only if $E$ and $F$ satisfy the same test sentences.

Proof. Trivially, if $E$ and $F$ are elementarily equivalent over $K$, then they satisfy the same test sentences. Conversely, assume that $E$ and $F$ satisfy the same test sentences, and let $E_{0}=E \cap \tilde{K}$ and $F_{0}=F \cap \tilde{K}$. By Lemma 10.6(3), $E_{0} \subseteq K^{\mathfrak{S}}$ and $F_{0} \subseteq K^{\mathfrak{S}}$. Let $f \in K[X]$ be an irreducible polynomial. If $f$ does not completely decompose over $K^{\mathfrak{S}}$, then it has no root in $K^{\mathfrak{S}}$, so it has no root in $E_{0}$ and it has no root in $F_{0}$. If $f$ completely decomposes over $K^{\mathfrak{S}}$, then $(\exists x)(f(x)=0)$ is a test sentence. Hence, $f$ has a root in $E_{0}$ if and only if it has a root in $F_{0}$. Therefore, $E_{0} \cong_{K} F_{0}$, see for example [FJ08, 20.6.3]. By Proposition 10.8, $E \equiv_{K} F$.

Lemma 11.9. The set of test sentences is recursive. 
Proof. Given a polynomial $f \in K[X]$, one can decide whether $(\exists x)(f(x)=0)$ is a test sentence because $N_{K}\left(K^{\mathfrak{S}}\right)$ is recursive by Lemma 11.5. Induction on the structure of formulas then shows that the set of test sentences is recursive.

Definition 11.10. For each $\mathcal{L}_{\text {ring }}(K)$-sentence $\theta$ let $\Sigma_{\mathfrak{S}, e}(\theta)=\left\{\boldsymbol{\sigma} \in \operatorname{Gal}(K)^{e}: K^{\mathfrak{S}}(\boldsymbol{\sigma}) \models \theta\right\}$. We denote by $\mu$ the unique Haar probability measure on $\operatorname{Gal}(K)^{e}$.

Lemma 11.11. Let $\lambda$ be a test sentence. Then $\Sigma_{\mathfrak{S}, e}(\lambda)$ is open-closed in $\operatorname{Gal}(K)^{e}$ and $\mu\left(\Sigma_{\mathfrak{S}, e}(\lambda)\right) \in \mathbb{Q}$. The map $\lambda \mapsto \mu\left(\Sigma_{\mathfrak{S}, e}(\lambda)\right)$ from test sentences to $\mathbb{Q}$ is recursive.

Proof. Let $f_{1}, \ldots, f_{n} \in K[X]$ be the polynomials occurring in $\lambda$. Their splitting field $L_{\lambda}$ is a finite Galois extension of $K$ inside $K^{\mathfrak{S}}$. Let $L / K$ be a Galois extension with $L_{\lambda} \subseteq L \subseteq K^{\mathfrak{S}}$ (this is needed for the induction). Then $K^{\mathfrak{S}}(\boldsymbol{\sigma}) \cap L=L\left(\operatorname{res}_{\tilde{K} / L}(\boldsymbol{\sigma})\right)$ for each $\boldsymbol{\sigma} \in \operatorname{Gal}(K)^{e}$. Let

$$
\Sigma_{L, \lambda}=\left\{\boldsymbol{\tau} \in \operatorname{Gal}(L / K)^{e}: L(\boldsymbol{\tau}) \models \lambda\right\} .
$$

We claim that $\Sigma_{\mathfrak{S}, e}(\lambda)=\left\{\boldsymbol{\sigma} \in \operatorname{Gal}(K)^{e}: \operatorname{res}_{\tilde{K} / L}(\boldsymbol{\sigma}) \in \Sigma_{L, \lambda}\right\}$. Indeed, if $\lambda$ is of the form $(\exists x)(f(x)=0)$, where $f \in K[X]$ completely decomposes over $K^{\mathfrak{S}}$, then

$$
\Sigma_{L, \lambda}=\left\{\boldsymbol{\tau} \in \operatorname{Gal}(L / K)^{e}: f \text { has a zero in } L(\boldsymbol{\tau})\right\} .
$$

Since $L$ contains all roots of $f, K^{\mathfrak{S}}(\boldsymbol{\sigma}) \models \lambda$ if and only if $K^{\mathfrak{S}}(\boldsymbol{\sigma}) \cap L \models \lambda$, so the claim is true in that case. Induction on the structure of $\lambda$ shows that the claim holds for all test sentences $\lambda$. Thus, $\Sigma_{\mathfrak{S}, e}(\lambda)$ is open-closed, in particular measurable. Furthermore,

$$
\mu\left(\Sigma_{\mathfrak{S}, e}(\lambda)\right)=\frac{\left|\Sigma_{L_{\lambda}, \lambda}\right|}{\left[L_{\lambda}: K\right]^{e}} \in \mathbb{Q}
$$

is computable since $K$ has a splitting algorithm, see for example [FJ08, 19.3.2].

Theorem 11.12. Under Setting 11.3, the following holds:

(1) For every $\mathcal{L}_{\text {ring }}(K)$-sentence $\theta, \Sigma_{\mathfrak{S}, e}(\theta)$ is $\mu$-measurable, and $\mu\left(\Sigma_{\mathfrak{S}, e}(\theta)\right) \in \mathbb{Q}$.

(2) The map $\theta \mapsto \mu\left(\Sigma_{\mathfrak{S}, e}(\theta)\right)$ from $\mathcal{L}_{\text {ring }}(K)$-sentences to $\mathbb{Q}$ is recursive.

In particular, the theory $T_{\text {almost, }, \text {,e }}$ of almost all fields $K^{\mathfrak{S}}(\boldsymbol{\sigma}), \boldsymbol{\sigma} \in \operatorname{Gal}(K)^{e}$, is decidable.

Proof. By Theorem 10.11, $T_{\text {tot, }, e} \models T_{\text {almost, }, e}$ and $T_{\text {almost, }, e, e} \models T_{\text {tot, }, e}$. By Lemma 11.8 and [FJ08, 7.8.2], for every $\mathcal{L}_{\text {ring }}(K)$-sentence $\theta$ there exists a test sentence $\lambda$ such that the sentence $\theta \leftrightarrow \lambda$ is in $T_{\text {almost, }, e, e}$. In particular, $\Sigma_{\mathfrak{S}, e}(\theta)$ and $\Sigma_{\mathfrak{S}, e}(\lambda)$ differ only by a zero set. Lemma 11.11 implies that $\Sigma_{\mathfrak{S}, e}(\lambda)$ is $\mu$-measurable and $\mu\left(\Sigma_{\mathfrak{S}, e}(\lambda)\right) \in \mathbb{Q}$, so also $\Sigma_{\mathfrak{S}, e}(\theta)$ is $\mu$-measurable and $\mu\left(\Sigma_{\mathfrak{S}, e}(\theta)\right)=\mu\left(\Sigma_{\mathfrak{S}, e}(\lambda)\right) \in \mathbb{Q}$.

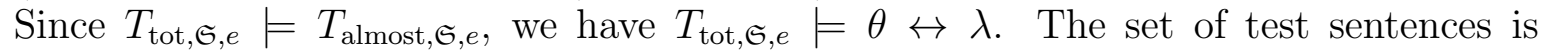
recursive by Lemma 11.9, By Lemma 11.6, the theory $T_{\text {tot, }, \mathfrak{S}_{e}}$ is recursive, so the set of consequences of $T_{\text {tot, }, e, e}$ is recursively enumerable. Therefore, there is a recursive map $\theta \mapsto \lambda_{\theta}$ from $\mathcal{L}_{\text {ring }}(K)$-sentences to test sentences such that $\theta \leftrightarrow \lambda_{\theta}$ is in $T_{\text {almost, }, \mathfrak{S}, e}$ for every $\theta$. In particular, $\mu\left(\Sigma_{\mathfrak{S}, e}(\theta)\right)=\mu\left(\Sigma_{\mathfrak{S}, e}\left(\lambda_{\theta}\right)\right)$. Since also the map $\lambda \mapsto \mu\left(\Sigma_{\mathfrak{S}, e}(\lambda)\right)$ from test sentences to $\mathbb{Q}$ is recursive by Lemma 11.11, so is the composition $\theta \mapsto \lambda_{\theta} \mapsto$ $\mu\left(\Sigma_{\mathfrak{S}, e}\left(\lambda_{\theta}\right)\right)=\mu\left(\Sigma_{\mathfrak{S}, e}(\theta)\right)$.

Remark 11.13. By applying this theorem to Example 11.4 we finally deduce Theorem 1.1 from the introduction.

Remark 11.14. Note that the assumption that the primes in $\mathfrak{S}$ are recursive is necessary. Indeed, $R_{\mathfrak{p}}\left(K^{\mathfrak{S}}\right)$ is $K$-definable in $K^{\mathfrak{S}}$ for each $\mathfrak{p} \in \mathfrak{S}$, cf. Proposition 7.12. An element 
$x \in K$ lies in $\mathcal{O}_{\mathfrak{p}}$ if and only if $x \in R_{\mathfrak{p}}\left(K^{\mathfrak{S}}\right)$, so the decidability of the complete $\mathcal{L}_{\text {ring }}(K)$ theory of $K^{\mathfrak{S}}$ implies that $\mathcal{O}_{\mathfrak{p}}$ is recursive. On the other hand we do not know whether the assumption that $K$ has a splitting algorithm is necessary.

The theorem does certainly not hold anymore if we allow $\mathfrak{S}$ to be an arbitrary (possibly infinite) set of recursive local primes of $K$. In fact, although there exist trivial examples of Hilbertian fields $K$ with an infinite set of local primes $\mathfrak{S}$ such that $K^{\mathfrak{S}}$ is decidable, we do not know any infinite set of primes $\mathfrak{S}$ of $K=\mathbb{Q}$ for which the theorem holds. Moreover, [Jar95, Example 10.4] gives an example of an infinite set of primes $\mathfrak{S}$ of $\mathbb{Q}$ that has Dirichlet density zero, but $\mathbb{Q}^{\mathfrak{S}}=\mathbb{Q}$, hence $T_{\text {almost }, \mathfrak{S}, e}=\operatorname{Th}(\mathbb{Q})$ is undecidable.

Remark 11.15. With the machinery developed here and some additional work one can show that Theorem 11.12 holds with $K^{\mathfrak{S}}(\boldsymbol{\sigma})$ replaced by the maximal Galois extension $K^{\mathfrak{S}}[\boldsymbol{\sigma}]$ of $K$ inside $K^{\mathfrak{S}}(\boldsymbol{\sigma})$. We refer the interested reader to [Feh10, Chapter 5], where this is shown for number fields $K$.

\section{Appendix A. Real Closed fields}

We recall the notion of real closed fields and quote some well known results from [Pre84.

Let $K$ be a field. A positive cone of $K$ is a semiring $P \subseteq K$ such that $P \cup(-P)=K$ and $P \cap(-P)=\{0\}$. An ordering of $K$ is a total order $\leq$ on $K$ such that $\{x \in K: x \geq 0\}$ is a positive cone. The map that assigns to an ordering the corresponding positive cone induces a natural bijection between the orderings of $K$ and the positive cones of $K$. An ordered field is a field $K$ together with an ordering. An ordering $\leq$ of $K$ is archimedean if for every $x \in K$ there exists $y \in \mathbb{N} \subseteq K$ with $x<y$. A pre-positive cone of $K$ is a semiring $P \subseteq K$ such that $K^{2} \subseteq P$ and $-1 \notin P$.

Lemma A.1. Each pre-positive cone of $K$ is the intersection of the positive cones of $K$ containing it. In particular, each pre-positive cone of $K$ is contained in a positive cone of $K$.

Proof. See [Pre84, 1.6].

A field is real closed if it has an ordering but each proper algebraic extension has no ordering. A real closed field $K$ has a unique ordering, given by the positive cone $K^{2}$, [Pre84, 3.2]. A real closed field $F$ is a real closure of an ordered field $K$ if $F$ is an algebraic extension of $K$ and the unique ordering of $F$ extends the ordering of $K$. Any ordered field $K$ has a real closure, which is unique up to $K$-isomorphism, [Pre84, 3.10]. If $L$ is a finite extension of an ordered field $K$, then the extensions of the ordering of $K$ to $L$ bijectively correspond to the $K$-embeddings of $L$ into a fixed real closure of $K$, Pre84, $3.12]$.

Lemma A.2. A field which is algebraically closed in a real closed field is real closed.

Proof. See [Pre84, 3.13].

Proposition A.3 (Artin-Schreier). A field $K$ is real closed if and only if $\operatorname{Gal}(K) \cong \mathbb{Z} / 2 \mathbb{Z}$.

Proof. This follows from [Lan02, VI.9.3] and [Pre84, 3.3].

Proposition A.4. Let $V$ be a smooth absolutely irreducible variety over a real closed field $K$. If $V(K) \neq \emptyset$, then $V(K)$ is Zariski-dense in $V$.

Proof. This follows for example from [Pre84, 7.10].

Proposition A.5 (Tarski). The $\mathcal{L}$-theory of real closed ordered fields is model complete.

Proof. See [Mar02, 3.3.15, 3.3.16]. 


\section{ApPendix B. p-ADICALly CLOSED FIELDS}

We recall the notion of $p$-adically closed fields and quote some well known results from [PR84] and some properties of the absolute Galois group of a $p$-adically closed field.

A valuation $v$ on a field $K$ of characteristic zero with residue field of characteristic $p>0$ and corresponding valuation ring $\mathcal{O}$ is a $p$-valuation of $p$-rank $d \in \mathbb{N}$ if $\operatorname{dim}_{\mathbb{F}_{p}} \mathcal{O} / p \mathcal{O}=d$. We also say that the valued field $(K, v)$ is a $p$-valued field.

The residue field $\bar{K}_{v}$ of a $p$-valued field $(K, v)$ is finite, and the value group $v\left(K^{\times}\right)$is discrete and $v(p) \in \mathbb{Z}$. If $e=v(p)$ and $f=\left[\bar{K}_{v}: \mathbb{F}_{p}\right]$, then $d=e f,[$ PR84, p. 15]. We call $(p, e, f)$ the type of $(K, v)$. Thus, if two $p$-valued fields have the same type, then they have the same $p$-rank. If $L / K$ is an extension of $p$-valued fields, then $L$ and $K$ have the same $p$-rank if and only if they have the same type. In that case, this type is also the type of each intermediate extension of $L / K$.

A $p$-valued field is $p$-adically closed if it has no proper $p$-valued algebraic extension of the same $p$-rank. Every $p$-adically closed valued field $(K, v)$ has a unique $p$-valuation, [PR84, 6.15]. We therefore also call $K p$-adically closed. A $p$-adic closure of a $p$-valued field $(K, v)$ is an algebraic extension of $(K, v)$ which is $p$-adically closed of the same $p$-rank as $(K, v)$. A $p$-valued field $(K, v)$ is $p$-adically closed if and only if it is Henselian and the value group $v\left(K^{\times}\right)$is a $\mathbb{Z}$-group, [PR84, 3.1]. Here, an ordered abelian group $\Gamma$ is a $\mathbb{Z}$-group if it is discrete and $(\Gamma: n \Gamma)=n$ for each $n \in \mathbb{N}$. Any $p$-valued field $(K, v)$ has a $p$-adic closure. A $p$-adic closure of $(K, v)$ is unique up to $K$-isomorphism if and only if $v\left(K^{\times}\right)$is a $\mathbb{Z}$-group, [PR84, 3.2].

Lemma B.1. If a field is algebraically closed in a p-adically closed field $K$, then it is $p$-adically closed of the same $p$-rank as $K$.

Proof. See [PR84, 3.4].

Proposition B.2. Let $V$ be a smooth absolutely irreducible variety over a p-adically closed field $K$. If $V(K) \neq \emptyset$, then $V(K)$ is Zariski-dense in $V$.

Proof. This follows for example from [PR84, 7.8].

Proposition B.3. The $\mathcal{L}$-theory of p-adically closed valued fields of $p$-rank $d$ is model complete.

Proof. See [PR84, 5.1, 5.2, 5.6, 5.4].

A $p$-adic field is the completion of a $p$-valued number field, i.e. a finite extension of $\hat{\mathbb{Q}}_{p}$. A $p$-adic field $F$ is $p$-adically closed of $p$-rank $\left[F: \hat{\mathbb{Q}}_{p}\right]$, [PR84, p. 21].

Lemma B.4. Every p-adically closed field is elementarily equivalent to a p-adic field.

Proof. Let $E$ be $p$-adically closed, and let $K=\tilde{\mathbb{Q}} \cap E, K_{0}=\tilde{\mathbb{Q}} \cap \hat{\mathbb{Q}}_{p}$. By Lemma B.1, $K$ is $p$-adically closed of the same $p$-rank as $E$, so $K_{0}=\tilde{\mathbb{Q}} \cap \hat{\mathbb{Q}}_{p} \subseteq K$. Then $\left[K: K_{0}\right]<\infty$, c.f. [PR84, 2.9], so $F:=K \hat{\mathbb{Q}}_{p}$ is a $p$-adic field. Since $K$ is algebraically closed in $F, K$ and $F$ have the same $p$-rank by Lemma B.1. Therefore, $E \equiv K \equiv F$ by model completeness (Proposition B.3).

Proposition B.5. Let $K$ be p-adically closed. Then $\operatorname{Gal}(K)$ is finitely generated and torsion-free.

Proof. First, if $K$ is a $p$-adic field, then $\operatorname{Gal}(K)$ is finitely generated and $\operatorname{cd}_{l}(\operatorname{Gal}(K))=2$ for every $l$, [NSW08, 7.4.1, 7.1.8(i)], so in particular it is torsion-free. Since every $p$ adically closed field $K$ is elementarily equivalent to a $p$-adic field $K_{0}$ (Lemma B.4), and 
$\operatorname{Gal}\left(K_{0}\right)$ is finitely generated, $\operatorname{Gal}(K) \cong \operatorname{Gal}\left(K_{0}\right)$ has all the asserted properties, cf. [FJ08, 20.4.6].

Proposition B.6 (Neukirch-Pop-Efrat-Koenigsmann). Let $K$ be p-adically closed, and let $L$ be a field. If $\operatorname{Gal}(K) \cong \operatorname{Gal}(L)$, then $L$ is p-adically closed of the same type as $K$.

Proof. By Lemma B.4, $K$ is elementarily equivalent to a $p$-adic field $K_{0}$, and $\operatorname{Gal}(K) \cong$ $\operatorname{Gal}\left(K_{0}\right)$ by Proposition B.5 and [FJ08, 20.4.6]. By [Koe95, Theorem 4.1], if $\operatorname{Gal}\left(K_{0}\right) \cong$ $\operatorname{Gal}(L)$, then $L$ is $p$-adically closed. $\operatorname{But~} \operatorname{Gal}(L)$ determines the type of $L$, see for example [JR79, Lemma 1].

\section{Appendix C. Model theory of absolute Galois groups}

We now review how to translate statements about the inverse system $S(\operatorname{Gal}(F))$ of the absolute Galois group of a field into statements about the field itself. Such a translation is claimed already in the unpublished [CDM82, but without proof. In fact, the careless reader might be tempted to assume that one can prove [CDM82, Lemma 17] by giving an honest interpretation of the $\omega$-sorted structure $S(\operatorname{Gal}(F))^{\omega}$ in $F$, but experts think that this is actually impossible. On the other hand, the abstract proof presented in Cha02 is complete, but does not allow to conclude that the translation is recursive. For this section, we fix $\mathfrak{S}=\emptyset$ and let $\mathcal{L}_{\mathrm{co}}=\mathcal{L}_{\mathrm{co}, \emptyset}$.

Definition C.1. A explained in [CDM82, §1] one can encode finite extensions of $F$ of degree $n$ by $n^{3}$-tuples $\mathbf{a} \in F^{n^{3}}$. We denote the extension defined by $\mathbf{a} \in F^{n^{3}}$ by $F_{\mathbf{a}}$. If $\mathbf{a}_{1} \in F^{n_{1}^{3}}$ and $\mathbf{a}_{2} \in F^{n_{2}^{3}}$ encode extensions of degree $n_{1}$ resp. $n_{2}$, then an $F$-embedding of $F_{\mathbf{a}_{1}}$ into $F_{\mathbf{a}_{2}}$ is encoded by an $n_{1} n_{2}$-tuple $\mathbf{b} \in F^{n_{1} n_{2}}$.

Definition C.2. An admissible sequence of length $\lambda \leq \omega$ and degrees $\left(n_{i}\right)_{i<\lambda}$ is a sequence $\mathbf{a}=\mathbf{a}_{1} \mathbf{a}_{2} \ldots$ of elements of $F$ such that for $i<\lambda$, the tuple $\mathbf{a}_{i}$ encodes

(1) a finite Galois extension $F_{\mathbf{a}, i}$ of $F$ of degree at most $n_{i}$,

(2) an automorphism $\sigma_{\mathbf{a}, i} \in \operatorname{Gal}\left(F_{\mathbf{a}, i} / F\right)$,

(3) a finite extension $F_{\mathbf{a}, i}^{*}$ of $F$,

(4) an embedding $\epsilon_{\mathbf{a}, i}: F_{\mathbf{a}, i} \rightarrow F_{\mathbf{a}, i}^{*}$,

(5) and for each $j=0, \ldots, i$ an embedding $\epsilon_{\mathbf{a}, j, i}: F_{\mathbf{a}, j}^{*} \rightarrow F_{\mathbf{a}, i}^{*}$,

such that $F_{\mathbf{a}, i}^{*}$ is the compositum of all $\epsilon_{\mathbf{a}, j, i}\left(\epsilon_{\mathbf{a}, j}\left(F_{\mathbf{a}, j}\right)\right), j \leq i$, and for $k \leq j \leq i$, $\epsilon_{\mathbf{a}, i, i}=\operatorname{id}_{F_{\mathbf{a}, i}^{*}}$ and $\epsilon_{\mathbf{a}, j, i} \circ \epsilon_{\mathbf{a}, k, j}=\epsilon_{\mathbf{a}, k, i}$.

We intentionally do not keep track of the size of the tuples in order to avoid an overload of notation. We will also handle finite and infinite sequences rather sloppily.

Lemma C.3. For each $\lambda<\omega$ and $\mathbf{n}=\left(n_{i}\right)_{i<\lambda}$ there is an $\mathcal{L}_{\text {ring }}$-formula $\alpha_{\lambda, \mathbf{n}}$ such that for a tuple a of elements of $F, F \models \alpha_{\lambda, \mathbf{n}}(\mathbf{a})$ iff $\mathbf{a}$ is admissible of length $\lambda$ and degrees $\mathbf{n}$.

Proof. This is clear, see also [CDM82, §1].

Definition C.4. Every $x \in S(\operatorname{Gal}(F))$ is of the form $g N$ for some open normal subgroup $N \triangleleft \operatorname{Gal}(F)$ and we denote by $F_{x}$ the finite extension of $F$ that is the fixed field of $N$, and by $\sigma_{x} \in \operatorname{Gal}\left(F_{x} / F\right)$ the automorphism corresponding to $x$ under the natural isomorphism $\operatorname{Gal}(F) / N \cong \operatorname{Gal}\left(F_{x} / F\right)$ induced by restriction.

Definition C.5. A sequence $\mathbf{x}=\left(x_{i}\right)_{i<\lambda}$ of elements of $S(\operatorname{Gal}(F))$ and an admissible sequence a of length $\lambda$ are compatible if there exist $F$-isomorphisms $\phi_{i}: F_{x_{0}} \cdots F_{x_{i}} \rightarrow$ $F_{\mathbf{a}, i}^{*}$ with $\phi_{i}\left(F_{x_{i}}\right)=\epsilon_{\mathbf{a}, i}\left(F_{\mathbf{a}, i}\right)-$ so $\phi_{i}$ induces an isomorphism $\tilde{\phi}_{i}: F_{x_{i}} \rightarrow F_{\mathbf{a}, i}-$ and $\tilde{\phi}_{i} \circ \sigma_{x_{i}}=\sigma_{\mathbf{a}, i} \circ \tilde{\phi}_{i}$ for all $i<\lambda$, such that for $j \leq i,\left.\phi_{i}\right|_{F_{x_{0}} \cdots F_{x_{j}}}=\epsilon_{\mathbf{a}, j, i} \circ \phi_{j}$. 
Lemma C.6. Let $\mathbf{x}$ and $\mathbf{a}$ be compatible sequences of length $\lambda<\omega$.

(a) For every $x_{\lambda} \in S(\operatorname{Gal}(F))$ there exists $\mathbf{a}^{\prime}$ such that $\mathbf{a a}^{\prime}$ is admissible of length $\lambda+1$ and $\left(x_{0}, \ldots, x_{\lambda}\right)$ and $\mathbf{a a}^{\prime}$ are compatible.

(b) For every $\mathbf{a}^{\prime}$ such that $\mathbf{a a}^{\prime}$ is admissible of length $\lambda+1$ there exists $x_{\lambda} \in S(\operatorname{Gal}(F))$ such that $\left(x_{0}, \ldots, x_{\lambda}\right)$ and $\mathbf{a a}^{\prime}$ are compatible.

Proof. Let $\phi_{i}: F_{x_{0}} \cdots F_{x_{i}} \rightarrow F_{\mathbf{a}, i}^{*}$ for $i<\lambda$ be as in Definition C.5.

(a) Choose $\mathbf{a}^{\prime}$ such that there are isomorphisms $\phi^{\prime}: F_{x_{\lambda}} \rightarrow F_{\mathbf{a a}^{\prime}, \lambda}$ and $\phi_{\lambda}: F_{x_{0}} \cdots F_{x_{\lambda}} \rightarrow$ $F_{\mathbf{a a}^{\prime}, \lambda}^{*}$, and $\epsilon_{\mathbf{a a}^{\prime}, \lambda}=\phi_{\lambda} \circ \phi^{\prime-1}, \epsilon_{\mathbf{a a}^{\prime}, i, \lambda}=\phi_{\lambda} \circ \phi_{i}^{-1}, \sigma_{\mathbf{a}, \lambda}=\phi^{\prime} \circ \sigma_{x_{\lambda}} \circ \phi^{\prime-1}$.

(b) Extend the isomorphism $\phi_{\lambda-1}^{-1}: F_{\mathbf{a}, \lambda-1}^{*} \rightarrow F_{x_{0}} \cdots F_{x_{\lambda-1}}$ to an embedding $\psi: F_{\mathbf{a a}^{\prime}, \lambda}^{*} \rightarrow$ $\left(F_{x_{0}} \cdots F_{x_{\lambda}}\right)^{\tilde{w}}$ with $\psi \circ \epsilon_{\mathbf{a a}^{\prime}, \lambda-1, \lambda}=\phi_{\lambda-1}^{-1}$, and choose $x_{\lambda}$ such that $F_{x_{\lambda}}=\psi\left(\epsilon_{\mathbf{a a}^{\prime}, \lambda}\left(F_{\mathbf{a a}^{\prime}, \lambda}\right)\right)$ and $\sigma_{x_{\lambda}}$ corresponds to $\sigma_{\mathbf{a a}^{\prime}, \lambda}$ under $\psi \circ \epsilon_{\mathbf{a a}^{\prime}, \lambda}$.

Definition C.7. We assign to each bounded $\mathcal{L}_{\mathrm{co}}$-formula $\varphi\left(v_{0}, \ldots, v_{\lambda-1}\right)$ and tuple $\mathbf{n}=$ $\left(n_{i}\right)_{i<\omega}$ an $\mathcal{L}_{\text {ring }}$-formula $\varphi_{\text {ring, } \mathbf{n}}(\mathbf{u})$, where $\mathbf{u}=\mathbf{u}_{0} \mathbf{u}_{1} \ldots$, as follows:

(1) If $\varphi$ is $v_{i} \leq v_{j}$, then $\varphi_{\text {ring,n }}$ expresses that $\alpha_{k+1, \mathbf{n}}(\mathbf{u})$, where $k=\max \{i, j\}$, and that $\epsilon_{\mathbf{u}, i, k}\left(\epsilon_{\mathbf{u}, i}\left(F_{\mathbf{u}, i}\right)\right) \supseteq \epsilon_{\mathbf{u}, j, k}\left(\epsilon_{\mathbf{u}, j}\left(F_{\mathbf{u}, j}\right)\right)$, so that $\epsilon_{\mathbf{u}}$ induces an embedding $F_{\mathbf{u}, j} \rightarrow F_{\mathbf{u}, i}$.

(2) If $\varphi$ is $v_{i} \sqsubseteq v_{j}$, then $\varphi_{\text {ring,n }}$ expresses that $\alpha_{k+1, \mathbf{n}}(\mathbf{u})$, where $k=\max \{i, j\}$, and that $\epsilon_{\mathbf{u}}$ induces an embedding $F_{\mathbf{u}, j} \rightarrow F_{\mathbf{u}, i}$, and, under this embedding, $\left.\sigma_{\mathbf{u}, i}\right|_{F_{\mathbf{u}, j}}=\sigma_{\mathbf{u}, j}$.

(3) If $\varphi$ is $P\left(v_{i_{1}}, v_{i_{2}}, v_{i_{3}}\right)$, then $\varphi_{\text {ring,n }}$ expresses that $\alpha_{k+1, \mathbf{n}}(\mathbf{u})$, where $k=\max \left\{i_{1}, i_{2}, i_{3}\right\}$, and that the three embeddings $\epsilon_{\mathbf{u}, i_{\nu}, k} \circ \epsilon_{\mathbf{u}, i_{\nu}}, \nu=1,2,3$, induce isomorphisms between the $F_{\mathbf{u}, i_{\nu}}$, and, under these isomorphisms, $\sigma_{\mathbf{u}, i_{1}} \circ \sigma_{\mathbf{u}, i_{2}}=\sigma_{\mathbf{u}, i_{3}}$.

(4) If $\varphi$ is $v_{i}=v_{j}$, then $\varphi_{\text {ring,n }}$ expresses that $\alpha_{k+1, \mathbf{n}}(\mathbf{u})$, where $k=\max \{i, j\}$, and that $\epsilon_{\mathbf{u}}$ induces an isomorphism $\phi: F_{\mathbf{u}, i} \rightarrow F_{\mathbf{u}, j}$, and $\phi \circ \sigma_{\mathbf{u}, i}=\sigma_{\mathbf{u}, j} \circ \phi$.

(5) If $\varphi$ is $v_{i} \in G_{n}$, then $\varphi_{\text {ring,n }}$ expresses that $\alpha_{i+1, \mathbf{n}}(\mathbf{u})$ and that $\left[F_{\mathbf{u}, i}: F\right] \leq n$.

(6) If $\varphi$ is of the form $\psi \wedge \eta$, then $\varphi_{\text {ring, } \mathbf{n}}$ is $\psi_{\text {ring, } \mathbf{n}} \wedge \eta_{\text {ring, } \mathbf{n}}$, and if $\varphi$ is of the form $\neg \psi$, then $\varphi_{\text {ring, } \mathbf{n}}$ is $\neg \psi_{\text {ring, } \mathbf{n}}$.

(7) If $\varphi$ is of the form $\left(\exists v_{i} \in G_{n}\right)(\psi)$ and $\lambda \geq i$ is minimal such that the free variables in $\psi$ are among $v_{0}, \ldots, v_{\lambda}$, then, after renumbering the variables we can assume that $i=\lambda$, and $\varphi_{\text {ring, } \mathbf{n}}(\mathbf{u})$ is

$$
\left(\exists \mathbf{u}^{\prime}\right)\left(\alpha_{\lambda+1, \mathbf{n}^{\prime}}\left(\mathbf{u} \mathbf{u}^{\prime}\right) \wedge \psi_{\text {ring, } \mathbf{n}^{\prime}}\left(\mathbf{u}_{0} \ldots \mathbf{u}_{\lambda-1} \mathbf{u}^{\prime}\right)\right),
$$

where $\mathbf{n}^{\prime}:=\left(n_{0}, \ldots, n_{\lambda-1}, n\right)$.

If $\Sigma$ is a ranked set of coformulas in the variables $\left(v_{i}\right)_{i<\lambda}$, we let $\mathbf{n}:=\mathbf{n}_{\Sigma}:=\left(n_{i}\right)_{i<\lambda}$ with $n_{i}$ minimal such that the formula $G_{n_{i}}\left(v_{i}\right)$ is in $\Sigma$, and let $\Sigma_{\text {ring }}$ consist of all $\alpha_{i, \mathbf{n}}$, for $i<\lambda$, and all $\varphi_{\text {ring,n }}$ where $\varphi \in \Sigma$.

Lemma C.8. Let $\mathbf{x}$, a be compatible sequences of length $\lambda \leq \omega$ and degrees $\mathbf{n}$ and let $\varphi\left(v_{0}, \ldots, v_{\lambda-1}\right)$ be a bounded $\mathcal{L}_{\mathrm{co}}$-formula. Then $S(\operatorname{Gal}(F)) \models \varphi(\mathbf{x})$ if and only if $F \models \varphi_{\text {ring, } \mathbf{n}}(\mathbf{a})$.

Proof. This is proven by case distinction according to Definition C.7: In case (1)-(5), the claim follows immediately from the compatibility. In case (6), the claim is trivial by induction. In case (7), the claim follows by induction and Lemma C.6.

Proposition C.9. Let $\Sigma$ be a ranked set of coformulas in the variables $v_{0}, v_{1}, \ldots$ Then $\Sigma$ is cosatisfied in $\mathrm{Gal}(F)$ if and only if $\Sigma_{\text {ring }}$ is satisfied in $F$.

Proof. Let $\mathbf{n}=\mathbf{n}_{\Sigma}$ as in Definition C.7.

If $\Sigma$ is cosatisfied in $\operatorname{Gal}(F)$ by $\mathbf{x}=\left(x_{0}, x_{1}, \ldots\right)$, then $\left[F_{x_{i}}: F\right] \leq n_{i}$ for all $i$. By applying Lemma C.6 iteratively we get an admissible sequence $\mathbf{a}$ of degrees $\mathbf{n}$ such that 
$\mathbf{x}$ and $\mathbf{a}$ are compatible. In particular, $F \models \alpha_{i, \mathbf{n}}(\mathbf{a})$ for every $i$. For every $\varphi \in \Sigma$, $S(\operatorname{Gal}(F)) \models \varphi(\mathbf{x})$ implies $F \models \varphi_{\text {ring,n }}(\mathbf{a})$ by Lemma C.8, so $\Sigma_{\text {ring }}$ is satisfied in $F$.

Conversely, if $\Sigma_{\text {ring }}$ is satisfied by a sequence $\mathbf{a}$, then, since $\Sigma_{\text {ring }}$ contains all $\alpha_{i, \mathbf{n}}$, a is admissible of degrees $\mathbf{n}$. Lemma C.6 gives a sequence $\mathbf{x}$ in $S(\operatorname{Gal}(F))$ such that $\mathbf{x}$ and a are compatible. For every $\varphi \in \Sigma, F \models \varphi_{\text {ring,n }}(\mathbf{a})$ implies that $S(\operatorname{Gal}(F)) \models \varphi(\mathbf{x})$ by Lemma C.8, so $\Sigma$ is satisfied in $F$.

Corollary C.10. There is a recursive map $\varphi \mapsto \varphi_{\text {ring }}$ from bounded $\mathcal{L}_{\mathrm{co}}$-sentences to

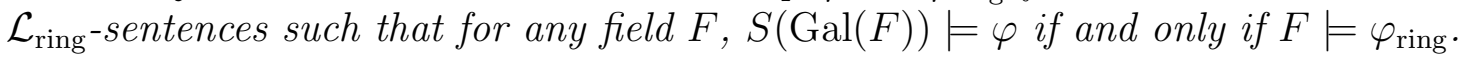

Proof. This is the special case $\Sigma=\{\varphi\}$ of Proposition C.9.

Corollary C.11. If $F$ is $\aleph_{1}$-saturated, then $\operatorname{Gal}(F)$ is $\aleph_{1}$-cosaturated.

Proof. Let $\Sigma$ be a countable ranked set of coformulas in the variables $v_{0}, v_{1}, \ldots$ with parameters in $S(\operatorname{Gal}(F))$ for which every finite subset is cosatisfied in $\operatorname{Gal}(F)$, and let $\mathbf{n}=\mathbf{n}_{\Sigma}$. For a finite subset

$$
\Sigma_{0}^{\prime}=\left\{\left(\varphi_{1}\right)_{\text {ring, }, \mathbf{n}}, \ldots,\left(\varphi_{m}\right)_{\text {ring, }, \mathbf{n}}, \alpha_{i_{1}, \mathbf{n}}, \ldots, \alpha_{i_{l}, \mathbf{n}}\right\} \subseteq \Sigma_{\text {ring }}
$$

choose $\lambda \geq \max \left\{i_{1}, \ldots, i_{l}\right\}$ such that the free variables of the $\varphi_{i}$ are among $v_{0}, \ldots, v_{\lambda}$, and let

$$
\Sigma_{0}:=\left\{\varphi_{1}, \ldots, \varphi_{m}, G_{n_{0}}\left(v_{0}\right), \ldots, G_{n_{\lambda}}\left(v_{\lambda}\right)\right\} .
$$

Since $\left(\varphi_{i}\right)_{\text {ring, } \mathbf{n}}=\left(\varphi_{i}\right)_{\text {ring, } \mathbf{n}_{\Sigma_{0}}}$ we see that $\Sigma_{0}^{\prime} \subseteq\left(\Sigma_{0}\right)_{\text {ring }}$, so since $\Sigma_{0}$ is cosatisfied in $\operatorname{Gal}(F)$, Proposition C.9 gives that $\left(\Sigma_{0}\right)_{\text {ring }}$, and hence $\Sigma_{0}^{\prime}$ is satisfied in $F$. Therefore, since $F$ is $\aleph_{1}$-saturated, $\Sigma_{\text {ring }}$ is satisfied in $F$, hence $\Sigma$ is cosatisfied in $\operatorname{Gal}(F)$, again by Proposition C.9.

\section{ACKNOWLEDGEMENTS}

The author would like to thank Zoé Chatzidakis and Franziska Jahnke for helpful discussions concerning the model theory of profinite groups. This work builds on Chapters 3 and 4 of the author's Ph.D. thesis [Feh10] supervised by Moshe Jarden at Tel Aviv University. As such, it was supported by the European Commission under contract MRTN-CT-2006-035495.

\section{REFERENCES}

[Cha84] Zoé Chatzidakis. Model Theory of Profinite Groups. PhD thesis, Yale University, 1984.

[Cha98] Zoé Chatzidakis. Model theory of profinite groups having the Iwasawa property. Illinois Journal of Mathematics, 42(1):70-96, 1998.

[Cha02] Zoé Chatzidakis. Properties of forking in $\omega$-free pseudo-algebraically closed fields. Journal of Symbolic Logic, 67(3):957-996, 2002.

[CDM81] Gregory Cherlin, Lou van den Dries, and Angus Macintyre. Decidability and undecidability theorems for PAC-fields. Bulletin of the American Mathematical Society, 4(1):101-104, 1981.

[CDM82] Gregory Cherlin, Lou van den Dries, and Angus Macintyre. The elementary theory of regularly closed fields. Manuscript, 1982.

[EP05] Antonio J. Engler and Alexander Prestel. Valued Fields. Springer, 2005.

[Ers91] Yuri Ershov. $P C_{p}$-fields with universal Galois group. Siberian Advances in Mathematics, 1(4):1-26, 1991.

[Ers95] Yuri Ershov. Fields with continuous local elementary properties. II. Algebra and Logic, 34(3):140-146, 1995.

[Ers96a] Yuri Ershov. Free $\Delta^{*}$-groups. Algebra and Logic, 35(2):86-95, 1996.

[Ers96b] Yuri Ershov. Nice local-global fields. I. Algebra and Logic, 35(4):229-235, 1996.

[Ers99] Yuri Ershov. Uniformly small $\Delta^{*}$-groups. Algebra and Logic, 38(1):12-20, 1999.

[Feh10] Arno Fehm. Decidability of large fields of algebraic numbers. PhD thesis, Tel Aviv University, 2010. 
[Feh13] Arno Fehm. Elementary geometric local-global principles for fields. Annals of Pure and Applied Logic, 164(10):989-1008, 2013.

[FHV94] Michael D. Fried, Dan Haran, and Helmut Völklein. Real Hilbertianity and the field of totally real numbers. In N. Childress and J. W. Jones, editors, Arithmetic Geometry, Contemporary Mathematics 174, pages 1-34. American Mathematical Society, 1994.

[FJ08] Michael D. Fried and Moshe Jarden. Field Arithmetic. Springer, third edition, 2008.

[Fro10] Nina Frohn. Model Theory of Absolute Galois Groups. PhD thesis, University of Freiburg (Breisgau), 2010.

[GJ02] Wulf-Dieter Geyer and Moshe Jarden. PSC Galois extensions of Hilbertian fields. Mathematische Nachrichten, 236(1):119-160, 2002.

[HJP09] Dan Haran, Moshe Jarden, and Florian Pop. The absolute Galois group of the field of totally S-adic numbers. Nagoya Mathematical Journal, 194:91-147, 2009.

[HJP12] Dan Haran, Moshe Jarden, and Florian Pop. The absolute Galois group of subfields of the field of totally $S$-adic numbers. Functiones et Approximatio Commentarii Mathematici, 2012.

[Jar95] Moshe Jarden. Totally $S$-adic extensions of Hilbertian fields. Manuscript, 1995.

[JK75] Moshe Jarden and Ursel Kiehne. The elementary theory of algebraic fields of finite corank. Inventiones Mathematicae, 30(3):275-294, 1975.

[JR79] Moshe Jarden and Jürgen Ritter. On the characterization of local fields by their absolute Galois groups. Journal of Number Theory, 11(1):1-13, 1979.

[JR98] Moshe Jarden and Aharon Razon. Rumely's local global principle for algebraic PSC fields over rings. Transactions of the American Mathematical Society, 350(1):55-85, 1998.

[Koe95] Jochen Koenigsmann. From p-rigid elements to valuations (with a Galois-characterization of p-adic fields). Journal für die reine und angewandte Mathematik, 465:165-182, 1995.

[Lan02] Serge Lang. Algebra. Springer, third edition, 2002.

[Mar02] David Marker. Model Theory: An Introduction. Springer, 2002.

[NSW08] Jürgen Neukirch, Alexander Schmidt, and Kay Wingberg. Cohomology of Number Fields. Springer, second edition, 2008.

[Pop86] Florian Pop. Galoissche Kennzeichnung p-adisch abgeschlossener Körper. PhD thesis, Heidelberg, 1986.

[PR84] Alexander Prestel and Peter Roquette. Formally p-adic Fields. Springer, 1984.

[Pre84] Alexander Prestel. Lectures on Formally Real Fields. Springer, 1984.

[Rum80] Robert Scott Rumely. Undecidability and definability for the theory of global fields. Transactions of the American Mathematical Society, 262(1):195-217, 1980.

[RZ00] Luis Ribes and Pavel Zalesskii. Profinite Groups. Springer, 2000.

[Shl07] Alexandra Shlapentokh. Hilbert's Tenth Problem: Diophantine Classes and Extensions to Global Fields. Cambridge University Press, 2007. 